\title{
A Kalman Filter Approach for Exploiting Bluetooth Traffic Data When Estimating Time-Dependent OD Matrices
}

\author{
J.Barceló, L.Montero, M.Bullejos, O. Serch and C. Carmona
}

Department of Statistics and Operations Research and CENIT (Center for Innovation in Transport) Technical University of Catalonia, Campus Nord, Jordi Girona, 1-3, Building C5, Office 215 - 08034 Barcelona, Spain

Phone: +34934017033

(jaume.barcelo, lidia.montero, manuel.bullejos, oriol.serch, carlos.carmona)@upc.edu

\begin{abstract}
Time-Dependent Origin-Destination (OD) matrices are essential input for Dynamic Traffic Models such as microscopic and mesoscopic traffic simulators. Dynamic traffic models also support real-time traffic management decisions and they are traditionally used in the design and evaluation of Traffic Management and Information Systems (ATMS/ATIS). Timedependent OD estimations are typically based either on Kalman-Filtering or on bi-level mathematical programming, which can be considered in most cases as ad hoc heuristics. The advent of the new Information and Communication Technologies (ICT) provides new types of traffic data with higher quality and accuracy, which in turn allows new modeling hypotheses that lead to more computationally efficient algorithms. This paper presents ad hoc, Kalman Filtering procedures that explicitly exploit Bluetooth sensor traffic data , and it reports the numerical results from computational experiments performed at a network test site.
\end{abstract}

Keywords: Time-Dependent Origin Destination Matrices, Estimation, Prediction, Kalman Filter, ICT, ATIS, ATMS

\section{INTRODUCTION}

The basic architectures of Advanced Traffic Information Systems (ATIS) and Advanced Traffic Management Systems (ATMS) share the same primary model components:

- A road network equipped with detection stations, which provide the data supporting the applications in a timely fashion.

- A Data Collection system collecting raw real-time traffic data that must be filtered and checked before being used by the models supporting the management system.

- An ad hoc Historic Traffic Database storing the traffic data used by traffic models in combination with the real-time data.

- Traffic models aimed at estimating and forecasting the short-term traffic state, fed with real-time and historic data.

- Time-Dependent, Origin-Destination (OD) matrices as inputs to Advanced Traffic Models. The estimation algorithms combine real-time and historic data along with other inputs which are not directly observable.

- Estimated and predicted states of the network that are compared with the expected states. If the comparison is OK, then no action is taken; otherwise, a decision is made (traffic policy) to achieve the desired objectives.

This paper addresses two main components of ATIS/ATMS: traffic data collection and the estimation of time-dependent Origin to Destination (OD) matrices (which are drawn from 
measurements of traffic variables). These are two of the many opportunities that new technologies provide transportation researchers and practitioners (Ran, Jin, Boyce, Qiu and Yang (2012)). Origin-Destination (OD) trip matrices, which describe traffic patterns across the network, are the primary data input used in traffic models and are therefore a critical requirement in all advanced systems that are supported by Dynamic Traffic Assignment models. However, because OD matrices are not directly observable, the current practice is to adjust an initial or seed matrix from link flow counts. which are provided by an existing layout of traffic counting stations. Information and Communication Technologies (ICT) applications provide new traffic measurements which allow more efficient algorithms for estimating OD matrices in real time. The OD matrices themselves are based on data exploited by modified Kalman Filtering. We assume the following: that inductive loop detectors collect the traffic data (i.e., volumes, occupancies and speeds); that new technologies are able to capture the electronic signature of specific on-board devices, such as a Bluetooth device; that these new technologies can complement the loop detectors with accurate measurements of travel times and speeds between two consecutive sensors. The objectives of this research are to investigate the data collection and filtering of Bluetooth measurements and to ascertain how these measurements can be computationally exploited by an ad hoc Kalman Filtering approach for estimating time-dependent OD matrices. This approach has been tested by simulation in two scenarios: freeways and urban networks. We have found that the quality of the estimations depends on various factors, such as: the penetration of the ICT devices, the detection layout and the quality of the initial information. Assuming that the decision on the Bluetooth layout is not already decided, we have proposed a model for laying out the sensors with the objective of getting high quality estimations. It is the same layout used in the computational experiments reported in the paper.

\section{DEALING WITH BLUETOOTH DATA}

As stated above, we assume that new technologies complement the usual approaches to traffic data collection, i.e., that sensors can now capture the public information from Bluetooth or Wi-Fi signals within its coverage radius. The most relevant information is the MAC address, whose singularity enables a matching algorithm to log the device when the sensor detects it. A vehicle equipped with a Bluetooth device traveling along the freeway is logged and timestamped at time $t_{1}$ by a sensor at location 1 . After traveling a certain distance it is logged and time-stamped again at time $t_{2}$ by another sensor downstream at location 2 . The difference in time stamps, $\tau=t_{2}-t_{1}$, measures the travel time of the vehicle equipped with that mobile device. The speed is also measured, assuming that the distance between both locations is known. Data captured by each sensor is sent to a central server by wireless telecommunications for processing. The basic principles on how these sensors operate are depicted in Figure 1.

Raw measured data cannot be used without previously filtering outliers that could bias the sample. The quality of the input data is crucial for the applications; therefore, it is a subject of intense research. A variety of nonlinear filters have been proposed recently by Van Lint and Hoogendoorn (2009) and Treiber, Kesting and Wilson (2011). A refined version of the Kalman Filter (Kalman, 1960), proposed in Barceló, Montero, Bullejos, Serch and Carmona (2010a) has been used in this paper. 


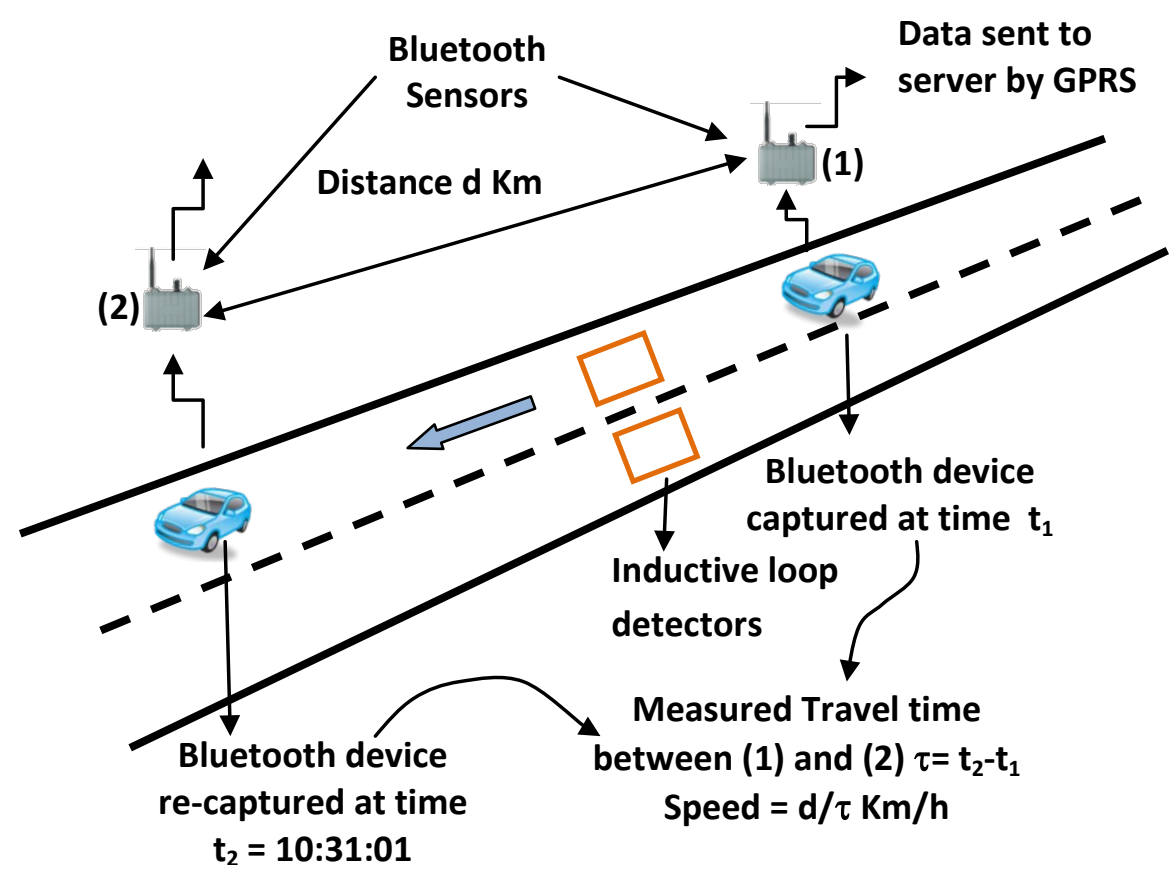

Figure 1. Vehicle monitoring with Bluetooth-based sensors

This clean data is the main input to a new Kalman Filter approach for estimating timedependent OD matrices. The proposed approach, which exploits the explicit travel time measurements from Bluetooth detectors, is based on a reformulation of the Kalman Filter approach for freeways explored in Barceló et al. (2010a). It also extends the approach to urban networks where alternative paths are available and route choice is relevant. This new approach exploits the measurements of travel times in order to reduce the number of state variables as well as to simplify the model. This paper is the follow-up to the preliminary work reported in Barceló, Montero, Bullejos, Serch and Carmona (2010b), and it provides conclusions based on computational experiments in an urban network test.

\section{A KALMAN APPROACH TO BLUETOOTH TRAFFIC DATA FILTERING}

We conducted a pilot project on a $40 \mathrm{Km}$. long section of the AP-7 Motorway, between Barcelona (Spain) and the French border. Figure 2 maps the area of the pilot project and highlights both the motorway length and the location of the sensors (colored circles), which are positioned on mile posts at Km. 87.2, 91.3, 106.4, 119.2, 125.4 and 130.5 of the AP-7 Motorway.

Figure 2 also depicts two examples of the measurements provided by the sensors at the borders of a motorway segment. The upper graphic corresponds to southward flow and the lower graphic to northward flow. The black line displays the time evolution of the speed between both locations throughout the day, and the blue area displays the quantity of detected devices. Table 1 shows an example of the raw data collected by the Bluetooth sensors and the filtered data used for forecasting. The (id) column identifies the temporal identity assigned by the encryption algorithm. Time1 and time2 identify, respectively, the time stamps. The final two columns correspond to the calculated speed and travel time. Data was collected over the two months of May and June 2009, and it was used to create a Historic Database of past measurements and traffic patterns which, together with real-time detection, provided the input for both the filtering and the forecasting algorithm (Barceló et al. 2010a). 


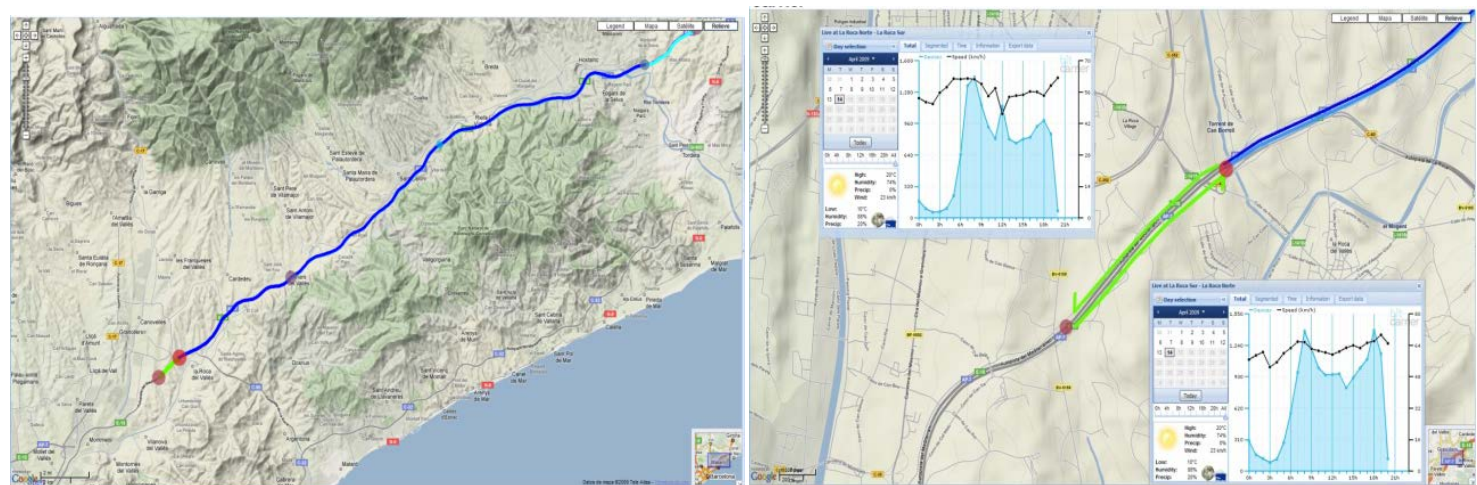

Figure 2. The site of the pilot project in the AP-7 Motorway in Barcelona and two examples of Bluetooth detection: speeds and quantity of detected devices

\begin{tabular}{|c|c|c|c|c|}
\hline id & time1 & time2 & km & $t_{2}-t_{1}$ (seconds) \\
\hline 10483 & 11/06/2009 19:07 & 11/06/2009 19:24 & 149,24 & 989 \\
\hline 11925 & 11/06/2009 18:29 & 11/06/2009 18:47 & 133,33 & 1107 \\
\hline 12660 & 11/06/2009 18:48 & 11/06/2009 19:06 & 134,92 & 1094 \\
\hline 18419 & 11/06/2009 17:18 & $11 / 06 / 2009$ 17:40 & 113,89 & 1296 \\
\hline 18613 & 11/06/2009 19:35 & 11/06/2009 19:53 & 136,16 & 1084 \\
\hline
\end{tabular}

Table 1. Example of raw measured travel times $\left(t_{2}-t_{1}\right)$ and speeds

The Kalman Filter (Kalman, 1960) is a State Space Model for estimating the dynamics of a system whose state at each instant $\mathrm{k}$ in time is defined by the values of a set of unobserved state variables, represented by a vector $\mathrm{x}(\mathrm{k}) \in \mathfrak{R}^{\mathrm{p}}$ (where $\mathrm{p}$ is the number of state variables). The system state transitions evolve over time, governed by the stochastic linear difference equation:

$$
\mathrm{x}(\mathrm{k})=\Phi \mathrm{x}(\mathrm{k}-1)+\mathrm{w}(\mathrm{k})
$$

where $\Phi$ is the transition matrix and $\mathrm{w}(\mathrm{k})$ represents the process noise --which is assumed to be white Gaussian-- with zero mean and covariance matrix $\mathrm{Q}$. The system is observed at time $\mathrm{k}$ with measurements $\mathrm{y}(\mathrm{k}) \in \mathfrak{R}^{\mathrm{q}}$ (where $\mathrm{q}$ is the number of observations) related to the state by the linear measurement equation:

$$
\mathrm{y}(\mathrm{k})=\mathrm{Ax}(\mathrm{k})+\mathrm{v}(\mathrm{k})
$$

where $\mathrm{A}$ is the measurement matrix, with a measurement noise $\mathrm{v}(\mathrm{k})$--also assumed to be white Gaussian-- with zero mean and covariance matrix R. Process and measurement noises are assumed to be independent, with covariance matrices $\mathrm{Q}$ and $\mathrm{R}$ respectively, which may change at every step. The discrete Kalman Filter cycles recursively between a time update, which projects the current state ahead in time from time step $\mathrm{k}-1$ to time step $\mathrm{k}$, and covariance estimateswith a similar time update. . This provides an a priori estimate of:

$$
\begin{aligned}
& \hat{\mathrm{x}}^{-}(\mathrm{k})=\Phi \hat{\mathrm{x}}(\mathrm{k}-1) \\
& \mathrm{P}_{\mathrm{k}}^{-}=\Phi \mathrm{P}_{\mathrm{k}-1} \Phi^{\mathrm{T}}+\mathrm{Q}
\end{aligned}
$$


It also provides a measurement update, which adjusts the projected estimate by using the available measurements at that time. The measurement update starts by computing the Kalman gain $\mathrm{G}_{\mathrm{k}}$, and then generates an a posteriori estimate by incorporating the measurements $y(k)$ at that time step and calculating the a posteriori error covariance estimate:

$$
\begin{aligned}
& G_{k}=P_{k}^{-} A^{T}\left(A P_{k}^{-} A^{T}+R\right)^{-1} \\
& \hat{x}(k)=\hat{x}^{-}(k)+G_{k}\left[y(k)-A \hat{x}^{-}(k)\right] \\
& P_{k}=\left(I-G_{k} A\right) P_{k}^{-}
\end{aligned}
$$

To illustrate this application of the Kalman Filter, we used the data collected from the pilot site on the AP-7 for a period of 16 weeks. Data were clustered by days of the week. In the example shown, we use the observations collected from the 16 Sundays in the sample. The data were divided into two groups: historical data (13 first Sundays) and test data (3 last Sundays).

After excluding days with incidents (4 days discarded), the historical data were used to: calibrate the input parameters of the Kalman Filter $\left(x_{0}, \Phi, P_{0}, Q, A, R\right)$, and to calculate the values of two statistics (Se, Sv)The first statistic (Se) contains the expected value of travel time and the second (Sv) its variability.

The value for each input parameter of the Kalman Filter was obtained from the statistics (Se, Sv) and the parameters were defined by the integer coefficients $(\alpha, \beta, \gamma)$.

$$
x_{0}=S e, \quad \Phi=1, \quad P_{0}=\frac{\sqrt{S v}}{\alpha}, \quad Q=\frac{\sqrt{S v}}{\beta}, \quad A=1, \quad R=\frac{\sqrt{S v}}{\gamma}
$$

The filtering process of travel time observations $\left(t t_{j}\right)$, applied on a test day $(t)$, used the predictions $\left(\hat{x}^{-}(k)\right)$ and their variance $\left(P_{k}^{-}\right)$, calculated by the Kalman Filter (3) at each step $(k)$ to select only valid observations $\left(\mathrm{OV}_{\mathrm{t}}^{\mathrm{k}}\right)$. From valid observations, the developed algorithm calculates the representative observation $(\mathrm{y}(\mathrm{k}))$ for the current step $(k)$ using the statistics (EST $\in\{$ mean, median, ... $\}$ ) applied to these observations.

$$
\begin{gathered}
\mathrm{OV}_{\mathrm{t}}^{\mathrm{k}}=\left\{t t_{j \in \mathrm{O}_{\mathrm{t}}^{\mathrm{k}}} \mid \hat{x}^{-}(k)+3 \cdot \sqrt{P_{k}^{-}} \geq t t_{j} \geq \hat{x}^{-}(k)-3 \cdot \sqrt{P_{k}^{-}}\right\} \\
y(k)=\operatorname{EST}\left(t t_{i \in \mathrm{O} \mathrm{V}_{\mathrm{t}}^{\mathrm{k}}}\right)
\end{gathered}
$$

$\mathrm{O}_{\mathrm{t}}^{\mathrm{k}}$ : set of observations for the test day $(t)$ obtained in the time interval $(k)$

The Kalman Filter uses the value of $(\mathrm{y}(\mathrm{k}))$ for calculating the current state $(\hat{x}(k))$ (4) which will be used to obtain predictions in the next step (3), continuing filtering process (see Figure 3).

The estimation of time arrivals to detectors is a main component of Kalman Filter estimates of OD matrices. This is usually based on dynamic traffic models of flow propagation, which account for nonlinearities, but it requires an Extended Kalman Filter. Lin and Chang (2007) simplify the model by using the flow dynamics to estimate a discrete travel time distribution; we propose further simplification by substituting the hypothesis on flow dynamics for the measurements provided by ICT sensors. The availability of clean filtered data, free of outliers, 
ensures the quality of the measurements for estimating the discrete travel time distribution used in the modified Kalman Filter Approach proposed in this paper. This is discussed in detail in the next section.

\section{DYNAMIC ESTIMATION OF OD MATRICES IN FREEWAYS, CORRIDORS AND NETWORKS}

The primary data input used in principal traffic models comes from Origin-Destination (OD) trip matrices, which describe the patterns of traffic behavior across the network. In this way, OD matrices become a critical requirement in Advanced Traffic Management and/or Information Systems that are supported by Dynamic Traffic Assignment models. However, OD matrices are not yet directly observable, even less so in the case of the time-dependent OD matrices that are necessary for Dynamic Traffic Assignment models; consequently, it has been natural to resort to indirect estimation methods. These indirect estimation methods are the so-called matrix adjustment methods, whose main modeling hypothesis can be stated as follows: if the flow of traffic in a network's links is the consequence of assigning an OD matrix to a network, then -given that we are capable of measuring link flows-- the problem of estimating the OD matrix that generates such flows can be considered the inverse of the assignment problem (Cascetta, 1984). Ever since the earliest formulation of the problem by Van Zuylen and Willumsen(1981), the matrix adjustment problem has been considered practical, as well as a relevant subject of research.

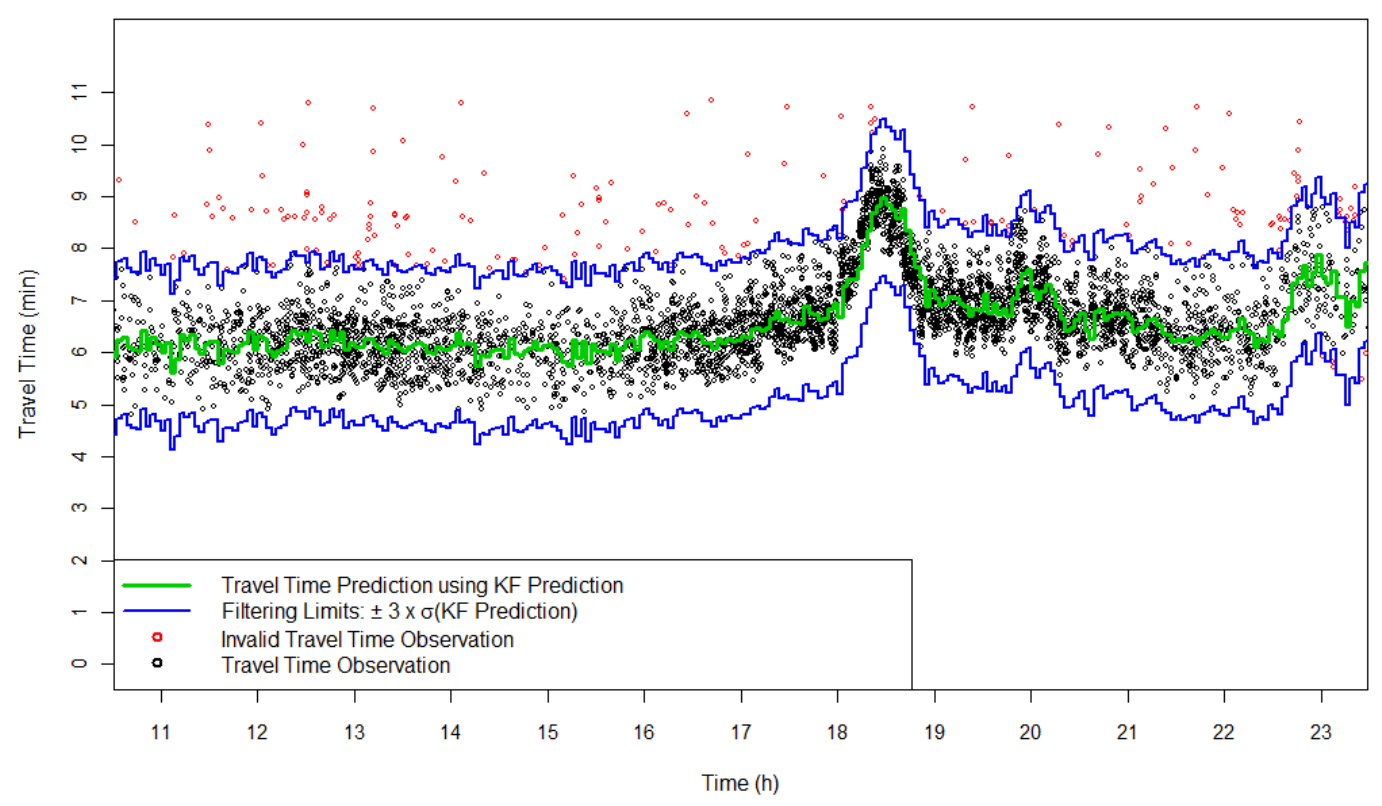

Figure 3. Kalman approach to Bluetooth travel times data filtering

Current practices consist of using an initial OD estimate and the OD seed or OD target as input. Then, they are adjusted from the available link counts provided by an existing layout of traffic counting stations and other additional information whenever it is available. Adjustments can be considered as indirect estimation methods, based either on discrete time optimization approaches (Codina \& Barceló (2004); Lundgren \& Peterson (2008)) or on adaptations of Kalman Filtering approaches (Ashok \& Ben Akiva, 2000; Antoniou, BenAkiva \& Koutsopoulos, 2007; Barceló et al. 2010a). 
The space-state formulations based on Kalman Filtering have always been an appealing approach for estimating time-dependent OD matrices. In this paper we propose a recursive linear Kalman-Filter for state variable estimation that combines and modifies the earlier works of Chang and $\mathrm{Wu}$ (1994); Hu, Madanat, Krogmeier and Peeta (2001); Choi, Jayakrishnan, Kim, Yang, and Lee (2009) and Van Der Zijpp and Hamerslag (1994). This adapts their models to take advantage of travel times and traffic counts collected by tracking Bluetooth-equipped vehicles and the use of other conventional detection technologies.

Chang and Wu (1994) proposed a model for freeways that, for each OD pair that estimates time-varying travel times, uses time-dependent traffic measures and implicit traffic flow models to account for flow propagation. The state variables are the time-varying OD proportions and the fractions of OD trips that arrive at each off-ramp $m$ interval after their entrance from on-ramps at interval $k$. The observation variables are main section and off-ramp counts for each interval. An Extended Kalman-filter approach is proposed for dealing with the nonlinear relationship between the state variables and the observations.

Hu et al. (2001) proposed an Extended Kalman Filtering algorithm for estimating dynamic OD matrices in which time-varying model parameters serve as state variables when formulating the model. The approach takes into account temporal issues of traffic dispersion. Lin and Chang (2007) proposed an extension of Chang and Wu (1994) in order to deal with traffic dynamics, assuming that travel time information is available.

Work, Toossavainen, Blandin, Bayen, Iwuchukwu and Tracton (2008) proposed using Ensemble Kalman Filtering as an algorithm for assimilating traffic data from active GPS mobile devices into a highway velocity model. Dixon and Rilett (2002) incorporated previously observed OD measurements with AVI data, as well as link counts, which improved the accuracy of OD estimations by basing them exclusively on link counts in a KF approach

For dynamic OD estimation, Zhou and Mahmassani (2006) proposed using a nonlinear ordinary least-squares estimation model to extract valuable point-to-point split-fraction information, which would come from automatic vehicle identification (AVI) counts. It would not estimate market-penetration rates or the identification rates of AVI tags.

We placed flow counting detectors and ICT sensors in a cordon and at each possible point for flow entry (centroids of the study area). ICT sensors were located at intersections in urban networks and covered access and links to/from the intersection. Flows and travel times were available from ICT sensors for any selected time interval higher than 1 second. Trip travel times from origin entry points to sensor locations are measurements provided by the detection layout. Therefore, they are no longer state variables but measurements, which simplify the model and make it more reliable.

A basic hypothesis is that equipped and non-equipped vehicles follow common OD patterns. We assume that this holds true in what follows and that it requires a statistical contrast for practical applications. Expansion factors from equipped vehicles to total vehicles, in a given interval, can be estimated by using the inverse of the proportion of ICT counts to total counts at centroids; expansion factors are assumed to be shared by all OD paths and pairs with a common origin centroid and initial interval. 
As a consequence of the experience gained in Barceló et al. (2010b), we propose a linear formulation of the Kalman Filtering approach that uses deviations of OD path flows as state variables, as suggested by (Ashok \& Ben-Akiva, 1993, 2000). It is calculated using the Dynamic User Equilibrium (DUE) Historic OD path flows for equipped vehicles. But our approach differs in that we do not require an assignment matrix. We use instead the subset of the most likely OD path flows identified from a DUE assignment with Dynameq (2010). The DUE is conducted with the historic OD flows, and the number of paths to take into account is a design parameter (a maximum of 10 paths per OD pair is currently considered, those 10 being the most important according to path flows across time-slices). DUE OD path proportions for selected paths and time-slices are not an input to the KF approach, only the description of the most likely OD paths. A list of paths going through each ICT sensor is automatically compiled from the OD path description, ICT sensor location and the network topology. In this way, once ICT sensor $j$ detects an equipped car, the travel time from its entry point to sensor $j$ is available and it is used for updating time varying model parameters that affect OD paths (state variables), which are included in the list.

We model the time-varying dependencies between measurements (sensor counts of equipped vehicles) and state variables (deviates of equipped OD path flows), adapting an idea of Lin and Chang (2010) for estimating discrete approximations to travel time distributions. The estimation of these distributions is made on the basis of flow models which induce nonlinear relationships that require extra state variables, leading to a nonlinear KF approach. Since our approach exploits the ICT travel time measurements from equipped vehicles, we can replace the nonlinear approximations with estimates from a sample of vehicles. This has advantages that constitute a major contribution of this paper:

- No extra state variables for modeling travel times and traffic dynamics are needed, since sampled travel times are used to estimate discrete travel time distribution (H bins are used for adaptive approximations). Also, nonlinear relationships involved in flow propagation can be avoided, thus making the Extended Kalman approach unnecessary.

- Travel times collected from ICT sensors are incorporated into the proposed model and it is not necessary that vehicles reach their destination, since at any intermediate sensor that they pass, the travel time between the entry point (centroid) to that sensor is used to update the discrete travel time approximation. Therefore, completed trips are not the only source for updating time-varying model parameters. No information about trajectories of equipped vehicles is used in this version. The use of sequential trajectory data from a given vehicle to define/identify paths and path travel time will be incorporated in the future.

In accordance with our experience, we agree with Antoniou et al. (2007) that formulations using deviations are beneficial to those using OD path flows as state variables, because they incorporate more historical data as a priori structural information for the model.

The demand matrix for the period under study is divided into several time-slices, which account for different proportions of the total number of trips in the time horizon. The approach assumes an extended space state variable for $\mathrm{M}+1$ sequential time intervals of equal length $\Delta \mathrm{t}$. $\mathrm{M}$ is the maximum number of time intervals required for vehicles to traverse the entire network in a congested scenario. The solution provides estimations of the OD matrices for each time interval up to the $k$-th interval. State variables $\Delta g_{i j c}(k)$ are deviations of OD path flows $g_{i j c}(k)$ relative to historic OD path flows $\tilde{g}_{i j c}(k)$ for equipped vehicles. If historic 
OD matrices are not available, then the formulation reduces to the case where state variables are the OD path flows. Total number of OD path flows $G_{i j c}(k)$ for all vehicles (equipped or not) are computed according to expansion factors $Q_{i}(k) / q_{i}(k)$ for each entry point and interval. OD path proportions are a post-processing result computed from the OD path flow deviations for equipped vehicles (state variables).

The total number of origin centroids is I, identified by index $i, i=1, . ., \mathrm{I}$; the total number of destination centroids $\mathrm{J}$, identified by index $j, j=1, \ldots, \mathrm{J}$; the total number of ICT sensors is Q, identified by index $q, q=1, \ldots \mathrm{Q}$ and the maximum number of most likely used paths between each origin and destination is $\mathrm{K}$. The notation used in this paper is the following:
$\tilde{Q}_{i}(k), \tilde{q}_{i}(k)$ : Historic total number of vehicles and equipped vehicles entering from centroid $i$ at time interval $k$
$Q_{i}(k), q_{i}(k) \quad$ : Total number of vehicles and equipped vehicles entering from centroid $i$ at time interval $\mathrm{k}$.
$\tilde{y}_{q}(k), y_{q}(k)$ : Historic and actual number of equipped vehicles crossing sensor $q$ at time interval $k$
$G_{i j c}(k), \quad \tilde{G}_{i j c}(k)$ : Total number of current $G_{i j c}(k)$ and historic $\tilde{G}_{i j c}(k)$ vehicles as well $g_{i j c}(k)^{\prime} \tilde{g}_{i j c}(k)$ as current $g_{i j c}(k)$ and historic $\tilde{g}_{i j c}(k)$ equipped vehicles entering the network from centroid $i$ at time interval $k$ headed towards destination $j$ using path $c$.

$\Delta g_{i j c}(k) \quad$ : State variables are deviates of equipped vehicles entering from centroid $i$ during interval $k$ headed towards centroid $j$ using path $c$ with respect to average historic flows $\Delta g_{i j c}(k)=g_{i j c}(k)-\tilde{g}_{i j c}$.

$\mathrm{z}(\mathrm{k}) \tilde{\mathrm{z}}(\mathrm{k}) \quad$ : The current and average historic measurements during interval $\mathrm{k}, \mathrm{a}$ column vector of dimension $\mathrm{Q}+\mathrm{I}$, whose structure is $z(k)^{T}=(y(k) \quad q(k))^{T}$

IJ, IJK : Number of feasible OD pairs and most likely OD paths depending on the zoning system defined in the network.

$u_{i q}^{h}(k) \quad:$ Fraction of vehicles that require $h$ time intervals to reach sensor $q$ at time interval $\mathrm{k}$ that entered the system from centroid $i$ (during time interval $[(k-h-1) \Delta t,(k-h) \Delta t])$.

$u_{i j c q}^{h}(k) \quad:$ Fraction of equipped vehicles detected at interval k whose trip from centroid $i$ to sensor $q$ might use OD path $(\mathrm{i}, \mathrm{j}, \mathrm{c})$ lasting $h$ time intervals of length $\Delta \mathrm{t}$ to arrive from centroid $i$ to sensor $q$, where $i=$ $1, \ldots, \mathrm{I}, j=1, \ldots, \mathrm{J}, h=1 \ldots M, q=1 . . Q Q$

$\bar{t}_{i q}(k) \quad:$ Average measured travel time for equipped vehicles entering at centroid $i$ and crossing sensor $q$ during interval $k$

$u_{i q}^{h}(k)$ and $u_{i j c q}^{h}(k)$ are the time-varying model parameters. The values of $u_{i q}^{h}(k)$ are provided by the measurements of the ICT sensors. The model also assumes that:

- For equipped vehicles, the entry points to the network and the time entering the network are known; thus $q_{i}(k)$ is also known. This accounts for trips traversing the scenario, trips starting outside and ending inside the scenario, starting inside and ending outside, or starting and ending at inner centroids. In these cases they are allocated to the centroid representing the zone where its associated ICT device has 
first been detected and counts have to be expanded by a factor that depends on the penetration of the ICT applications, unless other counts are available.

- Conservation equations from entry points (centroids) are explicitly considered.

- Without $Q_{i}(k)$, a generic expansion factor (defined as the inverse of the penetration rate) has to be applied to $g_{i j c}(k)$ to get $G_{i j c}(k)$. However, once $q_{i}(k)$ and $Q_{i}(k)$ are known --from the measurements $Q_{i}(k) / q_{i}(k)$-- a more realistic expansion factor is applied for all OD paths belonging to entry $i$.

Let $\Delta \mathrm{g}(\mathrm{k})$ be a column vector of dimension IJK containing the state variables $\Delta \mathrm{g}_{\mathrm{ijc}}(\mathrm{k})$ for each time interval $\mathrm{k}$ for all most likely OD paths (i,j,c). The state variables $\Delta \mathrm{g}_{\mathrm{ijc}}(\mathrm{k})$ are assumed to be stochastic in nature, and OD path flow deviates at current time $\mathrm{k}$ are related to the OD path flow deviates of previous time intervals by an autoregressive model of order $r$ $<<\mathrm{M}$; the state equations are:

$$
\begin{gathered}
\Delta \mathbf{g}(\mathbf{k}+\mathbf{1})=\sum_{\mathrm{w}=1}^{r} \mathrm{D}(\mathrm{w}) \Delta \mathrm{g}(\mathrm{k}-\mathrm{w}+1)+\mathrm{w}(\mathrm{k})=\mathbf{D} \Delta \mathbf{g}(\mathbf{k})+\mathbf{w}(\mathbf{k}) \\
\text { where } \quad \Delta \mathbf{g}(\mathbf{k})^{T}=\left(\begin{array}{llll}
\Delta \mathrm{g}(\mathrm{k}) & \Delta \mathrm{g}(\mathrm{k}-1) & \ldots & \Delta \mathrm{g}(\mathrm{k}-M)
\end{array}\right)^{T}
\end{gathered}
$$

And $\mathbf{w}_{\mathrm{ijc}}(\mathrm{k})$ 's are zero mean, while covariance matrix $\mathbf{W}_{\mathbf{k}}$, and $\mathbf{D}(\mathbf{w})$ are IJKxIJK transition matrices which describe the effects of previous OD path flow deviates $\Delta \mathrm{g}_{\mathrm{ijc}}(\mathrm{k}-\mathrm{w}+1)$ in current flows $\Delta \mathrm{g}_{\mathrm{ijc}}(\mathrm{k}+1)$ for $w=1, \ldots, r$. In this paper we assume simple random walks to provide the most flexible framework for state variables, since no convergence problems are detected. Thus when $r=1$, matrix $\mathbf{D}(\mathbf{w})$ is the identity matrix in the current implementation.

Two structures for the $\mathbf{W}_{\mathbf{k}}$ variance-covariance matrix of state variables have been considered: a multinomial structure and a diagonal. Multinomial structure at OD pair level means that for a given time interval $\mathrm{k}$ and OD pair with a common origin centroid, the covariance of the OD path flow behaves as counts in multinomial variables. When an OD path proportion increases, then the rest of the OD path flows (and thus proportions) for that OD pair decrease; i.e., OD path flows belonging to the same OD pair are related. Diagonal $\mathbf{W}_{\mathbf{k}}$ var-cov structures, the most used in applications reported in the literature, mean that state variables are considered statistically independent. The structural constraints to be satisfied by the state variables are:

$$
\begin{aligned}
& \Delta g_{i j c}(k) \geq-\tilde{g}_{i j c}(k) \quad i=1 \ldots I, \quad j=1 \ldots . . \quad c=1 \ldots . K_{i j}^{\max } \\
& q_{i}(k)=\sum_{j=1}^{J} \sum_{c=1}^{K_{i j}^{\max }} g_{i j c}(k) \rightarrow \sum_{j=1}^{J} \sum_{c=1}^{K_{i j}^{\max }} \Delta g_{i j c}(k)=q_{i}(k)-\tilde{q}_{i}(k) \quad i=1 \ldots I
\end{aligned}
$$

Equality constraints (6) are observation equations, as defined by dummy sums at centroids; measurement errors are allowed. The observation equations are sensor counts of the number of equipped vehicles entering the network for each interval $k$. The relationship between the state variables and the observations involves time-varying model parameters (congestiondependent, since they are updated from sample travel times provided by equipped vehicles) in a linear transformation that considers:

- The number of equipped vehicles entering from each entry centroid during time intervals $\mathrm{k}, \mathrm{k}-1, \mathrm{k}-\mathrm{M}, q_{i}(k)$.

- $\mathrm{H}<\mathrm{M}$ time-varying model parameters in form of fraction matrices, $\left[U_{i j c q}^{h}(k)\right]$. 
Structural constraints should also be satisfied by the time-varying model parameters $u_{i q}^{h}(k)$, reflecting temporal traffic dispersion and accounting for congestion, but are not explicitly considered as observation equations:

$$
\begin{gathered}
u_{i q}^{h}(k) \geq 0 \quad i=1 \ldots I, \quad q=1 \ldots Q, h=1 \ldots H \\
\sum_{h=1}^{H} u_{i q}^{h}(k)=1 \quad i=1 \ldots I, \quad, q=1 \ldots Q,
\end{gathered}
$$

Since time-varying model parameters are taken into account when modelling congestion, they are inferred from the $\mathrm{H}$ adaptive fractions that approximate $u_{i q}^{h}$. Measures provided by ICT sensors (conveniently filtered) are direct samples of travel times that allow updating discrete approximations of travel time distributions, making it unnecessary to incorporate models for traffic dynamics. This model simplification, due to the availability of the new ICT, is another major novelty in our proposed formulation. However, we must be aware that the final destination $j$ is unknown. Time-varying model parameters $u_{i q}^{h}$, which account for temporal traffic dispersion in affected paths $u_{i j c q}^{h}$, have to satisfy structural constraints, where $\mathrm{H}<\mathrm{M}$ :

$$
\begin{aligned}
& u_{i j c q}^{h}(k) \geq 0 \quad i=1 \ldots . . I, \quad j=1 \ldots . J, \quad c=1 \ldots . . K_{i j}^{\max }, \quad q=1, \ldots Q, \quad h=1 \ldots H \\
& \sum_{h=1}^{H} u_{i j c q}^{h}(k)=1 \quad i=1 \ldots . I, \quad j=1 \ldots . . J, \quad c=1 \ldots . K_{i j}^{\max }, \quad q=1, \ldots Q
\end{aligned}
$$

As in Lin and Chang (2007), we assume that the travel time $\mathrm{T}_{\mathrm{iq}}$ from origin $i$ to sensor $q$ is a random variable that depends on the evolution of traffic conditions, with a probability density function $t_{i q}^{(k)}(t)$ at interval $\mathrm{k}$. This can be approximated by the discrete travel time distribution for vehicles traveling $k$ from centroid $i$ to sensor $q$ at time interval $k$, i.e., in terms of $u_{i q}^{h}(k)$, updated from the (assumed random) sample of on-line travel time data of equipped vehicles (see Figure 4).

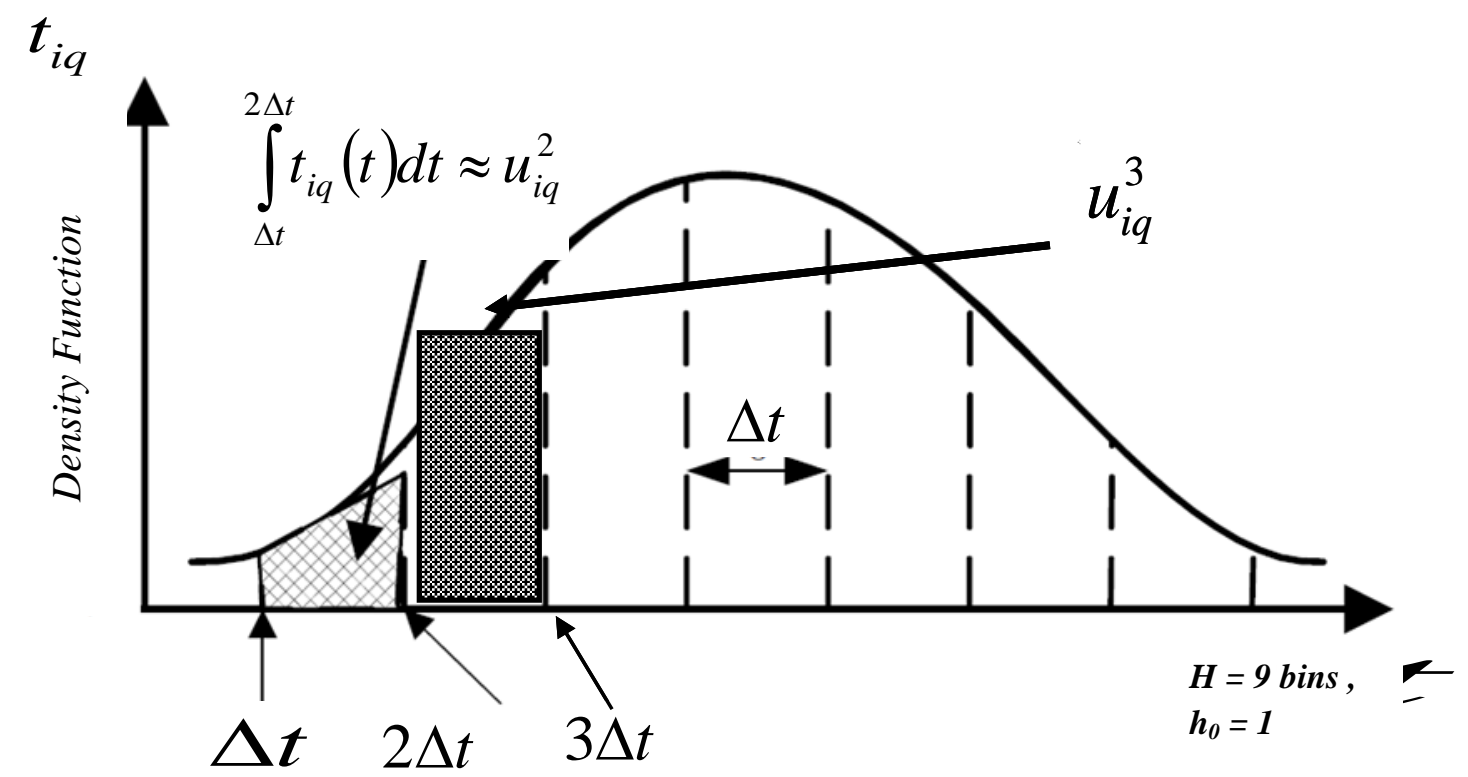

Figure 4. Discrete Approximation To Travel Time Distribution At Interval k.

A rolling horizon of $\mathrm{H}$ bins is kept updated; thus the mass probability is always divided into $\mathrm{H}$ consecutive interval bins of length $\Delta \mathrm{t}$. However, the first bin proportion belongs to a duration of $h_{0}$ intervals, the second to duration of $h_{0}+1, \ldots$ and the last one to duration $h_{0}+H-1$ time 
intervals, all of length $\Delta \mathrm{t}$. Initialization to $\mathrm{h}_{0}$, corresponding to free flow travel time, is set, and mass probability $u_{i q}^{h_{0}}(0)=1$ and $u_{i q}^{h_{0}+h}(0)=0 \quad h=2, \ldots, H-1$. As congestion increases, the approximations to travel time distributions adapt according to sampled data collected from equipped vehicles. Thus, $\mathrm{h}_{0}$ increases and the updated bin proportions are obtained by weighting former proportions and current sampled average travel time. This is one of the modeling contributions of this paper: the substitution of hypotheses on the dynamics of traffic flows with actual measures of travel times provided by ICT sensors. To formulate the model, we define the auxiliary matrices:

E(k) : Row matrix of dimension I containing 0 for columns related to state variables in time intervals $k-1, \ldots, k-M$ and defining conservation of flows (sum of OD path flows for each entry) at $k$.

$\mathbf{U}(\mathbf{k})$ : Consisting of diagonal matrices $U(k), \ldots, U(k-M)$ containing $u_{i j c q}^{h}(k) . U(k-h)$ is a matrix with the estimated proportion of equipped vehicles whose travel time from the access point to the network takes $h$ intervals and goes through the $q$ sensor at interval $k$.

A : Matrix that adds up sensor traffic flows from any possible entry, given timevarying model parameters at interval $k$.

At time interval $k$, the values of the observations are determined by those of the state variables at time intervals $k, k-1, \ldots k-M$.

$$
\Delta \mathrm{z}(\mathrm{k})=z(k)-\tilde{z}(k)=\left(\begin{array}{c}
y(\mathrm{k})-\tilde{y}(\mathrm{k}) \\
q(\mathrm{k})-\tilde{q}(\mathrm{k})
\end{array}\right)=\left(\begin{array}{c}
\mathbf{A U}(\mathbf{k})^{\mathbf{T}} \\
\mathbf{E}(\mathbf{k})
\end{array}\right) \Delta \mathbf{g}(\mathbf{k})+\left(\begin{array}{c}
\mathrm{v}_{1}(\mathrm{k}) \\
\mathrm{v}_{2}(\mathrm{k})
\end{array}\right)=\mathbf{F}(\mathbf{k}) \Delta(\mathbf{k}) \boldsymbol{\Delta}+\mathbf{v}(\mathbf{k})
$$

Where $\mathrm{v}_{\mathrm{i}}, \mathrm{i}=1,2$ are, respectively, white Gaussian noises with covariance matrices $\mathrm{R}_{\mathrm{i}} . \mathbf{F}(\mathbf{k})$ maps the state vector $\Delta \mathbf{g}(\mathbf{k})$ onto the current blocks of measurements at time interval $k$ : counts of equipped vehicles by sensors and entries at centroids, accounting for time lags and congestion effects. Deviate counts at $k$ mean the current observed counts minus the historical demand $\tilde{g}_{i j c}(k)$ counts, given the current traffic conditions (time-varying model parameters $\left.u_{i q}^{h}(k)\right)$.

To describe the model building process, we have chosen the example of the Hu, Peeta and Chu (2009) parallel highway network (Figure 5). The OD matrix consists of four non-zero OD flows $(1,8),(1,9),(2,8)$ and $(2,9)$ (identified as 1 to 4$)$. The whole set of OD paths is composed of routes described in Table 2 . BT sensors are assumed available at entry nodes 1 and 2, and links 6, 8, 9 and 10 (sensor ids are respectively 1 to 4).

\begin{tabular}{lll}
$\begin{array}{l}\text { OD } 6 \\
\text { min }\end{array}$ & $\mathbf{8}$ & $\mathbf{9}$ \\
\hline $\mathbf{1}$ & 120 & 120 \\
$\mathbf{2}$ & 40 & 40 \\
\hline
\end{tabular}

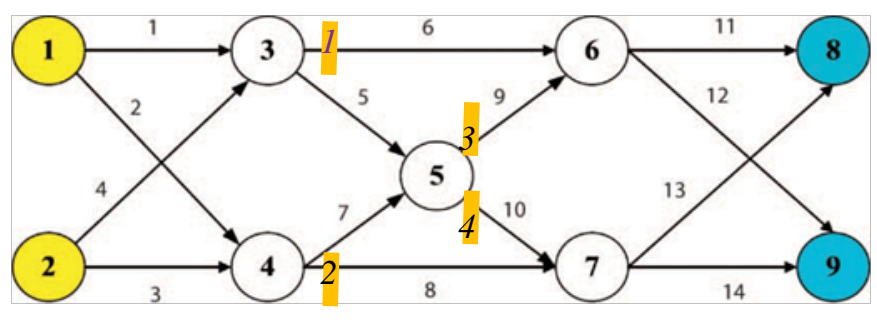

Figure 5 . Parallel highway network of Hu, Peeta and Chu (2009): link, BT sensors and node identifiers 


\begin{tabular}{|c|c|c|c|c|c|c|c|c|c|}
\hline $\begin{array}{l}\text { OD } \\
\text { Path/ } \\
\text { MLPath* } \\
\text { id }\end{array}$ & $\begin{array}{l}\text { OD path } \\
\text { links }\end{array}$ & $\begin{array}{l}\text { ML } \\
\text { Path id } \\
\text { in OD } \\
\text { pair }\end{array}$ & $\begin{array}{l}\text { OD } \\
\text { pair }\end{array}$ & $\begin{array}{l}\text { BT } \\
\text { id }\end{array}$ & $\begin{array}{l}\text { OD } \\
\text { path / } \\
\text { MLPath } \\
\text { id }\end{array}$ & $\begin{array}{l}\text { OD path } \\
\text { links }\end{array}$ & $\begin{array}{l}\text { ML } \\
\text { Path id } \\
\text { in OD } \\
\text { pair }\end{array}$ & $\begin{array}{l}\text { OD } \\
\text { pair }\end{array}$ & $\begin{array}{l}\text { BT } \\
\text { id }\end{array}$ \\
\hline $1 / 1$ & $1-6-11$ & 1 & $1=(1,8)$ & 1 & $13 / 7$ & $3-7-9-11$ & 1 & $3=(2,8)$ & 3 \\
\hline $2 / 2$ & $1-5-9-11$ & 2 & $1=(1,8)$ & 3 & $14 / 8$ & 3-7-10-13 & 2 & $3=(2,8)$ & 4 \\
\hline $3 / 3$ & $1-5-10-13$ & 3 & $1=(1,8)$ & 4 & $15 / 9$ & $3-8-13$ & 3 & $3=(2,8)$ & 2 \\
\hline 4 & 2-7-9-11 & & $1=(1,8)$ & 3 & 16 & $4-6-11$ & & $3=(2,8)$ & 1 \\
\hline 5 & $2-7-10-13$ & & $1=(1,8)$ & 4 & 17 & $4-5-9-11$ & & $3=(2,8)$ & 3 \\
\hline 6 & 2-8-13 & & $1=(1,8)$ & 2 & 18 & $4-5-10-13$ & & $3=(2,8)$ & 4 \\
\hline 7 & 1-6-12 & & $2=(1,9)$ & 1 & 19 & 3-8-14 & & $4=(2,9)$ & 2 \\
\hline 8 & $1-5-9-12$ & & $2=(1,9)$ & 3 & 20 & $3-7-10-14$ & & $4=(2,9)$ & 4 \\
\hline 9 & $1-5-10-14$ & & $2=(1,9)$ & 4 & 21 & $3-7-9-12$ & & $4=(2,9)$ & 3 \\
\hline $10 / 4$ & 2-8-14 & 1 & $2=(1,9)$ & 2 & $22 / 10$ & $4-5-10-14$ & 1 & $4=(2,9)$ & 4 \\
\hline $11 / 5$ & $2-7-10-14$ & 2 & $2=(1,9)$ & 4 & $23 / 11$ & 4-6-12 & 2 & $4=(2,9)$ & 1 \\
\hline $12 / 6$ & 2-7-9-12 & 3 & $2=(1,9)$ & 3 & 24/12 & 4-5-9-12 & 3 & $4=(2,9)$ & 3 \\
\hline
\end{tabular}

* MLPath means Most Likely path (according to DUE)

Table 2 OD path identifiers in the example

Let's assume that the set of most likely used paths is $\{1,2,3,10,11,12,13,14,15,22,23,24\}$. In this case $\mathrm{IJ}=4$ and $I J K=12$ (not 24 ). $\Delta \mathrm{t}=90 \mathrm{sec}$. To simplify we assume: that travel times for each link are $\Delta \mathrm{t}+\varepsilon$ for all links except link 1 , whose travel time is $2 \Delta \mathrm{t}+\varepsilon$ for all $\mathrm{k}>0$ subintervals; and that free-flow travel times are $\Delta \mathrm{t}+\varepsilon$ for all the links in $\mathrm{k}=0$. Time-varying model parameters are initialized $u_{i q}^{h_{0}}(0)$, as indicated in the fourth column in Table 3. Those values account for temporal traffic dispersion, and for each [i,q] entry pair $u_{i q}^{h}(k)$, they are applied to all most likely used OD paths linking entry i to sensor q. We also assume that the extended vector of states is the current one and that $\mathrm{M}$, the number of $\Delta$ t subintervals is set to $M=5$. Initially, the deviations of the most likely OD path flows are set equal to the historic OD path flows, and $100 \%$ of BT equipped vehicles is assumed; thus $\Delta g_{i j c}(0)=0$.

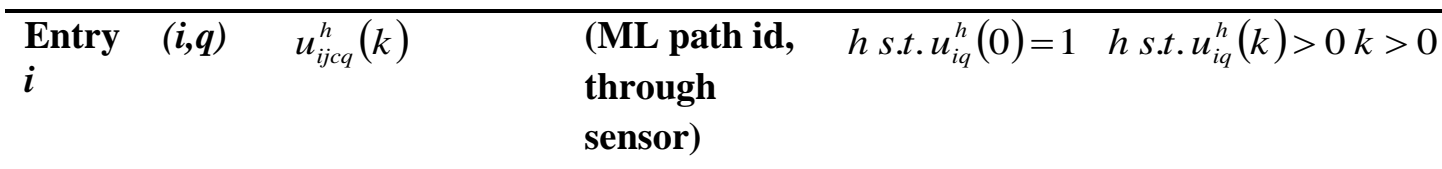

\begin{tabular}{llllll}
\hline $\mathbf{1}$ & {$[1,1]$} & $1-8-1-1$ & $(1,1)$ & 1 & $1,2\left(u_{11}^{1}(k)+u_{11}^{2}(k)=1\right)$ \\
$\mathbf{1}$ & {$[1,2]$} & $1-9-1-2$ & $(4,2)$ & 1 & $1\left(u_{12}^{1}(k)=1\right)$ \\
$\mathbf{1}$ & {$[1,3]$} & $1-8-2-3,1-9-3-3$ & $(2,3)(6,3)$ & 2 & $2,3\left(u_{13}^{2}(k)+u_{13}^{3}(k)=1\right)$ \\
$\mathbf{1}$ & {$[1,4]$} & $1-8-3-4,1-9-2-4$ & $(3,4)(5,4)$ & 2 & $2,3\left(u_{14}^{2}(k)+u_{14}^{3}(k)=1\right)$ \\
$\mathbf{2}$ & {$[2,1]$} & $2-9-2-1$ & $(11,1)$ & 1 & $1\left(u_{21}^{1}(k)=1\right)$ \\
$\mathbf{2}$ & {$[2,2]$} & $2-8-3-2$ & $(9,2)$ & 1 & $1\left(u_{22}^{1}(k)=1\right)$ \\
$\mathbf{2}$ & {$[2,3]$} & $2-8-1-3,2-9-3-3$ & $(7,3),(12,3)$ & 2 & $2\left(u_{23}^{2}(k)=2\right)$ \\
$\mathbf{2}$ & {$[2,4]$} & $2-8-2-4,2-9-1-4$ & $(8,4),(10,4)$ & 2 & $2\left(u_{24}^{2}(k)=2\right)$ \\
\hline
\end{tabular}

Table 3. Values for time-varying model parameters $u_{i j c q}^{h}(k)$ and $u_{i q}^{h}(k)$ 
Let $\Delta \mathbf{g}(\mathbf{k})$ be a column containing state variables for intervals $k, k-1, \ldots, k-M$ of dimension $(\mathrm{M}+1) \times I J K=(5+1) \times 12=72$.

The extended vector state has $\operatorname{IJKx}(\mathrm{M}+1)=12 \times 6=72$ components in the example and, for $\mathrm{r}=1$, the state variable equations are defined as a random walk which can be expressed for the extended state vector as a shifting operator (equation 5) by setting D as a $72 \times 72$ matrix with non-null submatrix components $\mathbf{I}_{\mathbf{1 2}}$ (identity matrix 12x12).

$$
\mathbf{D}=\left(\begin{array}{cccc}
\mathbf{I}_{12} & 0 & \ldots & 0 \\
\mathbf{I}_{12} & 0 & 0 & 0 \\
0 & \ddots & 0 & 0 \\
0 & 0 & \mathbf{I}_{12} & 0
\end{array}\right)
$$

The time-varying linear operator relates state variables and current observations for time interval $k$ in $\mathrm{Eq}(9)$. In the example $\Delta \mathrm{z}(\mathrm{k})$ is a vector of dimension $(Q+I)=4+2=6$. A discretization of the travel-time distribution in $\mathrm{H}=2$ bins is enough in this case (the first bin $h_{0}$ is represented by the free flow travel times). Then, if $C_{i}$ is the number of most likely used paths originating in the network at entry $i$, for $i=1, \ldots, I$ and $\sum_{i=1, \ldots, I} C_{i}=I J K$, then $C_{1}=6$ and $\mathrm{C}_{2}=6$ in our case and $\mathbf{E}(\mathbf{k})$ in Eq. (9) results in:

$$
\mathbf{B}=\left(\begin{array}{cc}
\overbrace{111111}^{C_{1}=6} & \overbrace{111111}^{C_{2}=6}
\end{array}\right), \quad \mathbf{E}=\left[\begin{array}{llllll}
\mathbf{B} & \mathbf{0}_{I J K} & \mathbf{0}_{I J K} & \mathbf{0}_{I J K} & \mathbf{0}_{I J K} & \mathbf{0}_{I J K}
\end{array}\right]
$$

A matrix in Eq. (9) is composed by appending identity matrices of dimension Q, M+1=5+1=6 times; i.e.:

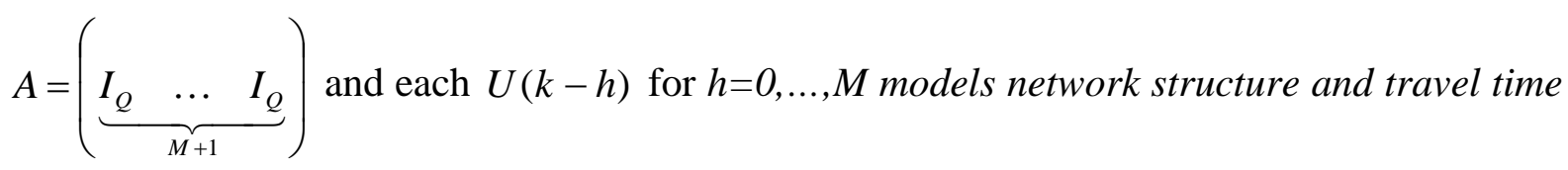
delays in terms of fractions on travel time bins (time-varying model parameters). Implementation guarantees that implicit structural restrictions are satisfied .

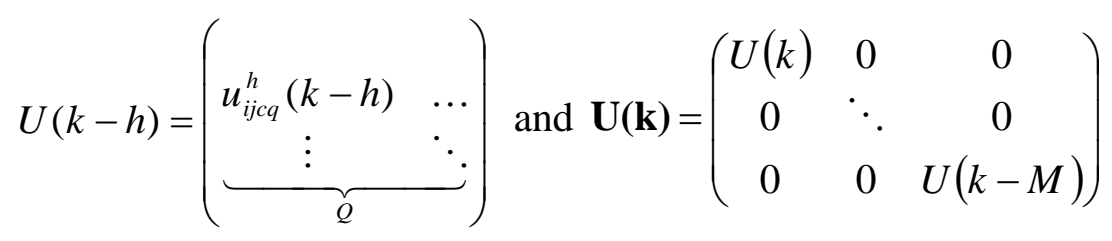

$\mathbf{U}(\mathbf{k})$ is $(1+\mathrm{M}) \operatorname{IJK} \times(1+\mathrm{M}) \mathrm{Q}=6 * 12 \times 6 * 4=72 \times 24$. In the example $u_{i j c q}^{h}(k-h)>0$ is the fraction of the equipped vehicles that entered the network $h$ intervals before $k$ at centroid $i$ that might reach sensor $q$ at $k$ interval (this fraction applies to all OD paths $(i, j, c)$ ). For $k=1$, submatrices in $\mathbf{U}(\mathbf{1})$ are defined as $U(1)=U(0)=U(-1)=U(-2)=U(-3)=U(-4)=\mathbf{0}_{12 \times 4}$ since vehicles that enter the system at subinterval $\mathrm{k}=1$ cannot reach any sensor. 
For $\mathrm{k}>\mathrm{M}$

$U(k)=\mathbf{0}_{12 \times 4} U(k-1)=\left(\begin{array}{cccc}0.5 & 0 & 0 & 0 \\ 0 & 0 & 0 & 0 \\ 0 & 0 & 0 & 0 \\ 0 & 1 & 0 & 0 \\ 0 & 0 & 0 & 0 \\ 0 & 0 & 0 & 0 \\ 0 & 0 & 0 & 0 \\ 0 & 0 & 0 & 0 \\ 0 & 1 & 0 & 0 \\ 0 & 0 & 0 & 0 \\ 1 & 0 & 0 & 0 \\ 0 & 0 & 0 & 0\end{array}\right) U(k-2)=\left(\begin{array}{cccc}0.5 & 0 & 0 & 0 \\ 0 & 0 & 0.5 & 0 \\ 0 & 0 & 0 & 0.5 \\ 0 & 0 & 0 & 0 \\ 0 & 0 & 0 & 0.5 \\ 0 & 0 & 0.5 & 0 \\ 0 & 0 & 1 & 0 \\ 0 & 0 & 0 & 1 \\ 0 & 0 & 0 & 0 \\ 0 & 0 & 0 & 1 \\ 0 & 0 & 0 & 0 \\ 0 & 0 & 1 & 0\end{array}\right) U(k-3)=\left(\begin{array}{cccc}0 & 0 & 0 & 0 \\ 0 & 0 & 0.5 & 0 \\ 0 & 0 & 0 & 0.5 \\ 0 & 0 & 0 & 0 \\ 0 & 0 & 0 & 0.5 \\ 0 & 0 & 0.5 & 0 \\ 0 & 0 & 0 & 0 \\ 0 & 0 & 0 & 0 \\ 0 & 0 & 0 & 0 \\ 0 & 0 & 0 & 0 \\ 0 & 0 & 0 & 0 \\ 0 & 0 & 0 & 0\end{array}\right)$

$U(k-4)=U(k-5)=\mathbf{0}_{12 \times 4}$

Finally, the algorithm can then be described as follows:

\begin{tabular}{|c|c|}
\hline $\begin{array}{l}\text { KF } \\
\text { Algorithm }\end{array}$ & $\begin{array}{l}\text { : Let } \mathrm{K} \text { be the total number of time intervals for estimation purposes, and } \\
\mathrm{M} \text { the maximum number of time intervals for the longest trip. }\end{array}$ \\
\hline Initialization & $\begin{array}{l}\text { k }=0 \text {; Build constant matrices and vectors: } \mathbf{A}, \mathbf{B}, \mathbf{D}, \mathbf{E} \text {. } \\
\left.\text { Inicialize } \mathbf{Q}_{\mathbf{k}} \text { (and } \mathbf{W}_{\mathbf{k}}\right), \mathbf{R}_{\mathbf{k}} \\
\begin{array}{l}\Delta \mathbf{g}_{\mathbf{k}}^{\mathbf{k}}=\Delta \mathbf{g}(\mathbf{0}) \text { where each time interval is set to the assumed historic OD } \\
\text { path flow } \\
\mathbf{P}_{\mathbf{k}}^{\mathbf{k}}=\mathbf{V}[\mathbf{w}(\mathbf{0})]\end{array}\end{array}$ \\
\hline $\begin{array}{l}\text { Prediction } \\
\text { Step }\end{array}$ & $\begin{array}{ll}: & \Delta \mathbf{g}_{\mathbf{k}+1}^{\mathbf{k}}=\mathbf{D} \Delta \mathbf{g}_{\mathbf{k}}^{\mathbf{k}} \\
& \mathbf{P}_{\mathbf{k}+1}^{\mathbf{k}}=\mathbf{D} \mathbf{P}_{\mathbf{k}}^{\mathbf{k}} \mathbf{D}^{\mathrm{T}}+\mathbf{W}_{\mathbf{k}}\end{array}$ \\
\hline $\begin{array}{l}\text { Kalman gain } \\
\text { computation }\end{array}$ & $\begin{array}{l}\text { Get observations of counts and travel times for BT equipped vehicles } \\
\text { and update fractions in travel time approximation bins: } \\
q(k+1), \quad y(k+1), \quad u_{i q}^{h}(k+1) \\
\text { Build } \Delta \mathrm{z}(\mathrm{k}+1) \text { and } \mathbf{U}(\mathbf{k}+\mathbf{1}) \\
\text { Build } \mathbf{F}_{\mathbf{k}+\mathbf{1}}=\mathbf{F}(\mathbf{k}+\mathbf{1})\end{array}$ \\
\hline & Compute Kalman gain $\mathbf{K}_{\mathbf{k}+\mathbf{1}}=\mathbf{P}_{\mathbf{k}+\mathbf{1}}^{\mathbf{k}} \mathbf{F}_{\mathbf{k}+\mathbf{1}}^{\mathbf{T}}\left(\mathbf{F}_{\mathbf{k}+\mathbf{1}} \mathbf{P}_{\mathbf{k}+\mathbf{1}}^{\mathbf{k}} \mathbf{F}_{\mathbf{k}+\mathbf{1}}^{\mathbf{T}}+\mathbf{R}_{\mathbf{k}}\right)^{-}$ \\
\hline Filtering & $\begin{array}{l}\text { Compute } \mathbf{d}_{\mathbf{k}+\mathbf{1}}=\mathbf{K}_{\mathbf{k}+\mathbf{1}}\left(\Delta \mathrm{z}(\mathrm{k}+1)-\mathbf{F}_{\mathbf{k}+\mathbf{1}} \Delta \mathbf{g}_{\mathbf{k}+\mathbf{1}}^{\mathbf{k}}\right) \text { filter for state variables and } \\
\text { errors } \boldsymbol{\varepsilon}_{\mathbf{k}+\mathbf{1}}=\left(\Delta z(k+1)-\mathbf{F}_{\mathbf{k}+\mathbf{1}} \Delta \mathbf{g}_{\mathbf{k}+\mathbf{1}}^{\mathbf{k}}\right) \\
\text { Search maximum step length } 0 \leq \alpha \leq 1 \text { such that } \\
\Delta g_{\mathbf{k}+\mathbf{1}}^{\mathbf{k}+\mathbf{1}}=\Delta g_{\mathbf{k}+1}^{\mathbf{k}}+\alpha \mathbf{d}_{\mathbf{k}+\mathbf{1}} \geq-\varepsilon \\
\mathbf{P}_{\mathbf{k}+\mathbf{1}}^{\mathbf{k}+\mathbf{1}}=\left(\mathbf{I}-\mathbf{K}_{\mathbf{k}+\mathbf{1}} \mathbf{F}_{\mathbf{k}+\mathbf{1}}\right) \mathbf{P}_{\mathbf{k}+\mathbf{1}}^{\mathbf{k}}\end{array}$ \\
\hline $\begin{array}{l}\text { Iteration } \\
\text { Exit }\end{array}$ & $\begin{array}{ll}: & k=k+1 \\
& \text { if } k=\mathrm{K} \text { EXIT otherwise GOTO Prediction Step } \\
: & \text { Print results }\end{array}$ \\
\hline
\end{tabular}




\section{APPLICATION TO NETWORKS: AMARA SIMULATION SITE}

Amara District (location?) is an urban network with 232 links, 142 nodes and 85 OD pairs. It has a rich structure of alternative paths between OD pairs, totaling 358 paths for the selected demand matrix, according to the DUE with (Dynameq, 2010). Figure 6 (a) displays a snapshot of the microsimulation model used to emulate Bluetooth data collection. Highlighted in red dots, Figure 6 (b) displays the detection layout of the 48 ICT detectors.

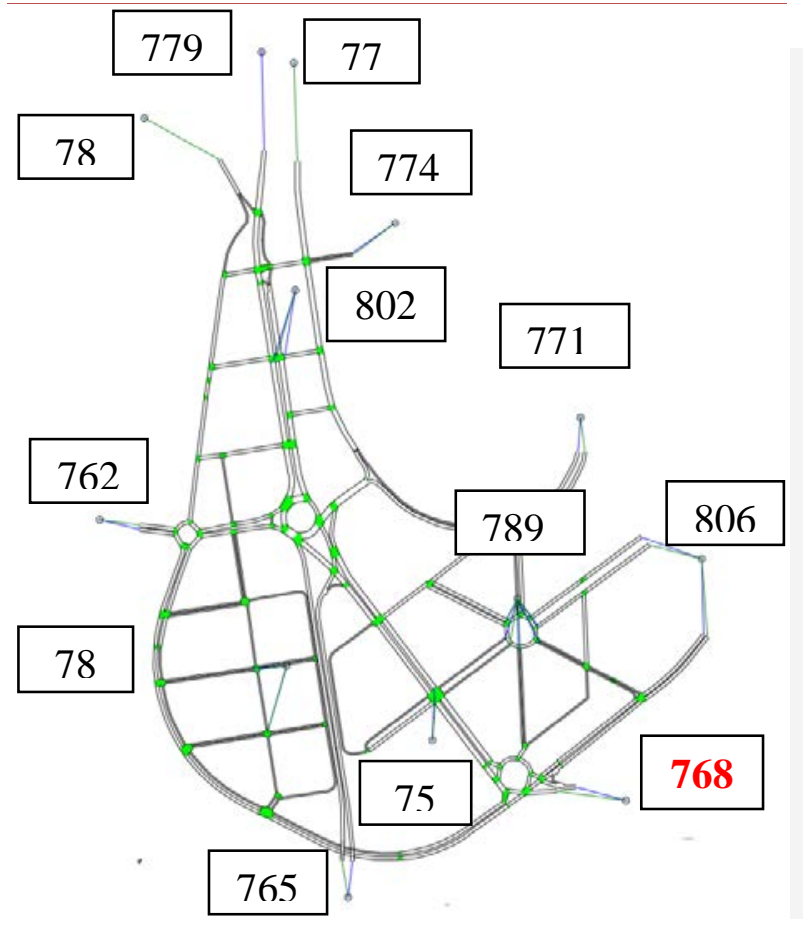

Figure 6. (a) Microsimulation Model: AMARA Test Site

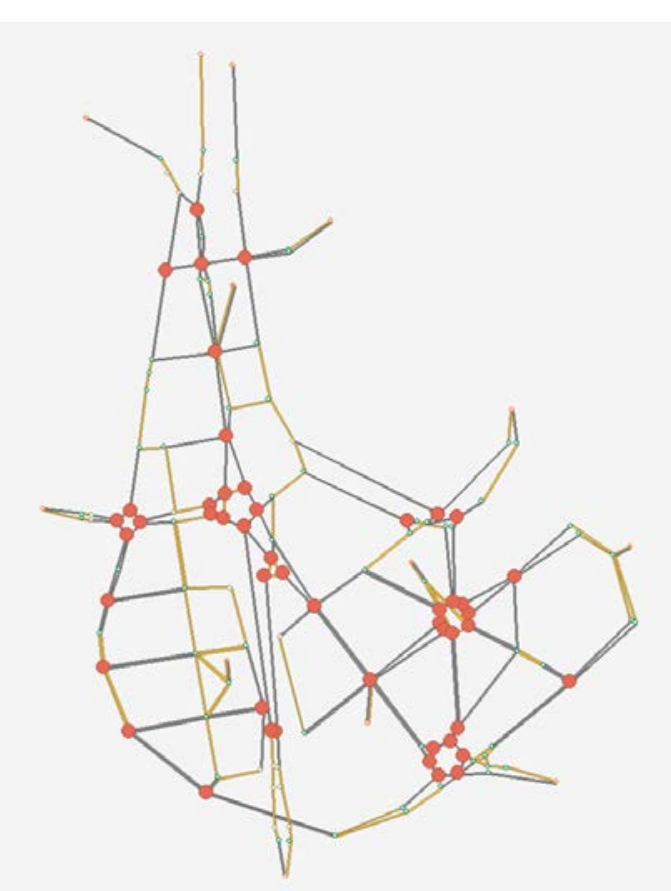

(b) Detection Layout

\section{Determining the Detection Layout}

The detection layout raises some methodological concerns. As we have specified, it consists of two components: the cordon component encircling the network with sensors at input-output gates (currently available in most of the urban pricing systems), and the detection layout at the interior of the encircled area. For estimating time-dependent OD matrices, the optimal sensor location is a problem strongly related to the observability of the network. Castillo, Conejo, Menéndez and Jiménez (2008) define the observability problem as the problem of identifying whether a set of available measurements is sufficient for estimating the state of a system. In the case of a state representation approach, this means that --for any possible sequence of state and control vectors-- the current system state (and therefore, the system behavior) can be determined using only the system measurements. Castillo et al. (2008) reformulate the problem for traffic networks in terms of "determining if a set of OD pair and link flows is sufficient to estimate the state of the network".

Yang and Zhou (1998) proposed a formulation of the problem in terms of a set covering model with additional constraints, which is still considered the main reference. They formulate the model on the basis of four modeling hypotheses or basic rules that must be satisfied by any optimal covering: 
1. O/D Covering rule: The counting stations on the road network should be located so that a certain proportion of trips between any O/D pair will be observed.

2. Maximal Flow Fraction rule: For a particular O/D pair, the counting stations on the road network should be located at the links so that the flow fraction between this O/D pair overflowing on these links is as large as possible.

3. Maximal Flow Intercepting rule: Under a certain number of links to be observed, the chosen links should intercept as many flows as possible.

4. Link Independence rule: The counting stations should be located on a network so that the resultant traffic counts on all chosen links are not linearly dependent.

This approach was tested for link flow data, measured by loop detectors in (Gilliéron and Barceló, 2008). However, when dealing with sensors capturing the electronic signature --such as the detectors of Bluetooth devices on board vehicles-- the detectors' location requires a new approach which takes into account that these detectors are more efficiently located at intersections than at links; this allows them to capture a higher number of vehicles. Let's analyze the scheme in Figure 7, assuming that the Bluetooth sensor is located at the intersection in a location in such a way that its detection lobule intercepts all equipped vehicles crossing the node on paths (1), (2), (3) and (4). The candidate intersections would be those intercepting a higher number of equipped vehicles.

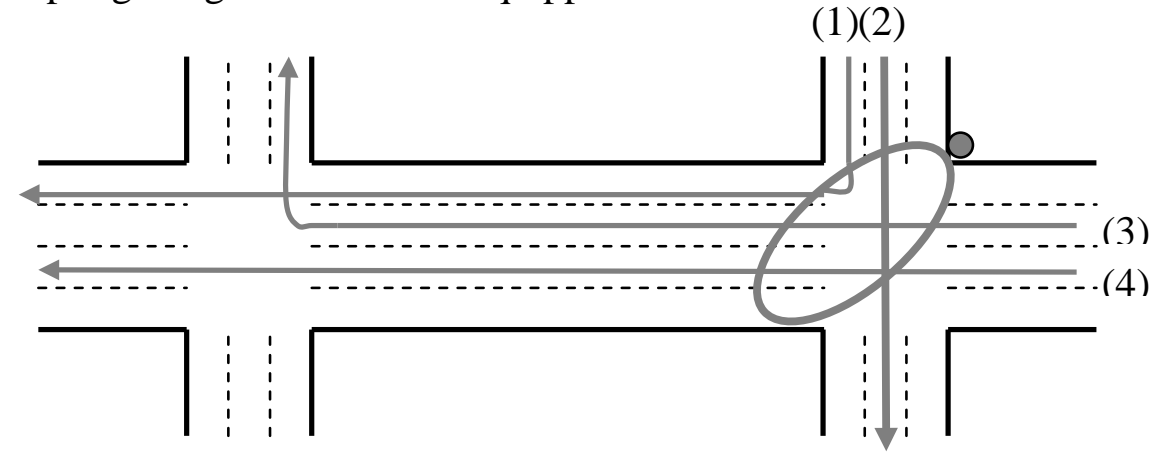

Figure 7. Flows intercepted from paths crossing a node

Following the same four modeling hypotheses proposed in the formulation of the link detection layout problem, and as a consequence of Rule 3 (Maximal Flow Intercepting Rule), a primary formulation of the intersection layout problem is the following. Let:

$$
\begin{aligned}
& x_{n}= \begin{cases}1 & \text { if a sensor is located at intersection } n \\
0 & \text { otherwise }\end{cases} \\
& y_{k}= \begin{cases}1 & \text { if there is at least one detector along path } k \\
0 & \text { otherwise }\end{cases} \\
& \delta_{n k}= \begin{cases}1 & \text { if intersection } n \text { is into path } k \\
0 & \text { otherwise }\end{cases} \\
& \hat{l}=\text { maximum number of detectors to be located } \\
& K=\text { set of all paths between all OD pairs }
\end{aligned}
$$


If path $k$ carries flow $h_{k}$, then $\sum_{k \in K_{n}} h_{k}$ is the total flow captured by the detector at node $\mathrm{n}$. $K_{n}$ is the set of paths crossing node n. Based on the experience with the previous link covering models, the problem is formulated in terms of the most likely used paths determined by the solution of a Dynamic User Equilibrium Assignment (Dynameq, 2010; Florian, Mahut \& Tremblay, 2001). We would be interested in identifying which is the configuration of nodes that maximizes the total flow intercepted by the sensors located there; therefore, we formulate the objective function in terms of Rule 3 by representing the total intercepted flow. To enhance the OD covering, we impose the condition that at least one path of each OD pair has a detector located on it; and for practical reasons we also include a bounding constraint on the maximum number $\hat{l}$ of detectors that can be located in a network. The problem can then be formulated in terms of the following extended set covering model with side constraints:

$$
\begin{aligned}
& \operatorname{MAX} \sum_{k \in K} h_{k} \cdot y_{k} \\
& \text { s.t. } \\
& \sum_{n \in N} x_{n} \leq \hat{\boldsymbol{l}} \\
& \sum_{n \in N} \delta_{n k} \cdot x_{n} \geq y_{k}, \quad \forall k \in K_{i}, \forall i \in I \\
& \sum_{k \in K_{i}} y_{k} \geq 1, \quad \forall i \in I \\
& \boldsymbol{x}_{\boldsymbol{n},} \boldsymbol{y}_{\boldsymbol{k}} \in\{\mathbf{0}, \mathbf{1}\}
\end{aligned}
$$

In this formulation the problem can be infeasible if $\hat{l}<l_{0}$, where $l_{0}$ is the minimum number of detectors necessary to satisfy the OD covering constraints. Therefore, we quantify the infeasibility when it appears. The location of Bluetooth sensors can raise additional questions, in terms of the measurement of travel times between pairs of detectors along the likely used paths, as has been described in the introduction of this section. In order to achieve this objective, we propose a new formulation of the model that adds two sets of constraints:

- Ensuring a minimum number of detectors on each path, and

- Imposing a condition of minimum linear distance between two detectors. This constraint can also be justified from the technological point of view of minimizing the likelihood of improper detection due to signal overlapping.

The new formulation would then be (11): 


$$
\begin{gathered}
\operatorname{MAX} \sum_{\substack{k \in K \\
\text { s.t. }}} h_{k} \cdot y_{k} \\
\sum_{n \in N} x_{n} \leq \hat{l} \\
\sum_{n \in N} \delta_{n k} \cdot x_{n} \geq p y_{k}, \quad \forall k \in K_{i}, \forall i \in I(*) \\
\sum_{k \in K_{i}} y_{k} \geq 1, \quad \forall i \in I \\
x_{i}+x_{j} \leq 1 \quad \forall i, \forall j \in V(i) \\
x_{n,} y_{k} \in\{0,1\}
\end{gathered}
$$

here:

- $\quad V(i)$ is the linear neighborhood of intersection $\mathrm{i}$, defined as:

$$
V(i)=\{j \in I \mid \operatorname{dist}(i, j) \leq m \text { meters }\}
$$

- $\quad p$ is the parameter defining the minimum number of detectors per path; constraints $\left.{ }^{*}\right)$ provide path information; $p>1$ provides travel times between detectors along a path

- $m$ is the minimum linear distance between two detectors

This node covering formulation of the detection layout problem has been developed and tested in Barceló, Gilliéron, Linares, Serch and Montero (2012). This has been the method of determining Amara's layout, depicted in Figure 6.

\section{DESIGN OF COMPUTATIONAL EXPERIMENTS}

Once a vehicle is generated in the simulation model, it is randomly identified as an equipped vehicle according to the design proportions of technological penetration in computational tests. The simulation emulates the logging and time stamping of this random sample of equipped vehicles. In the preliminary computational experiments conducted to test the KF approach, we focused our attention on the quality of the results and not on computational efficiency. A prototype has been implemented in MATLAB (Identified as KFX in what follows) and tested computationally. The length of the time interval has been set at 90 seconds and the simulation period to 1 hour.

The simulation experiments for testing the proposed approach have been conducted in the Amara network using a dynamic OD matrix with four time slices as input, corresponding to a rise in congested conditions. This OD, considered the true historical OD matrix, has been determined through simulation by building the Macro Fundamental Diagram (MFD) for the network (Daganzo \& Geroliminis, 2008), as shown in Figure 8. Therefore, congestion is not a design factor in these experiments. This OD is sliced into four 15-minute slices --each one 
accounting for $15 \%, 25 \%, 35 \%$ and $25 \%$ of the total number of trips-- to emulate demand variability. The simulation experiments with Aimsun use the described OD matrix, emulating traffic detection, and provide the inputs to the KFX model in terms of flow counts for ordinary and ICT sensors, as well as travel times from entry cordon $i$ to $q$ ICT sensor.

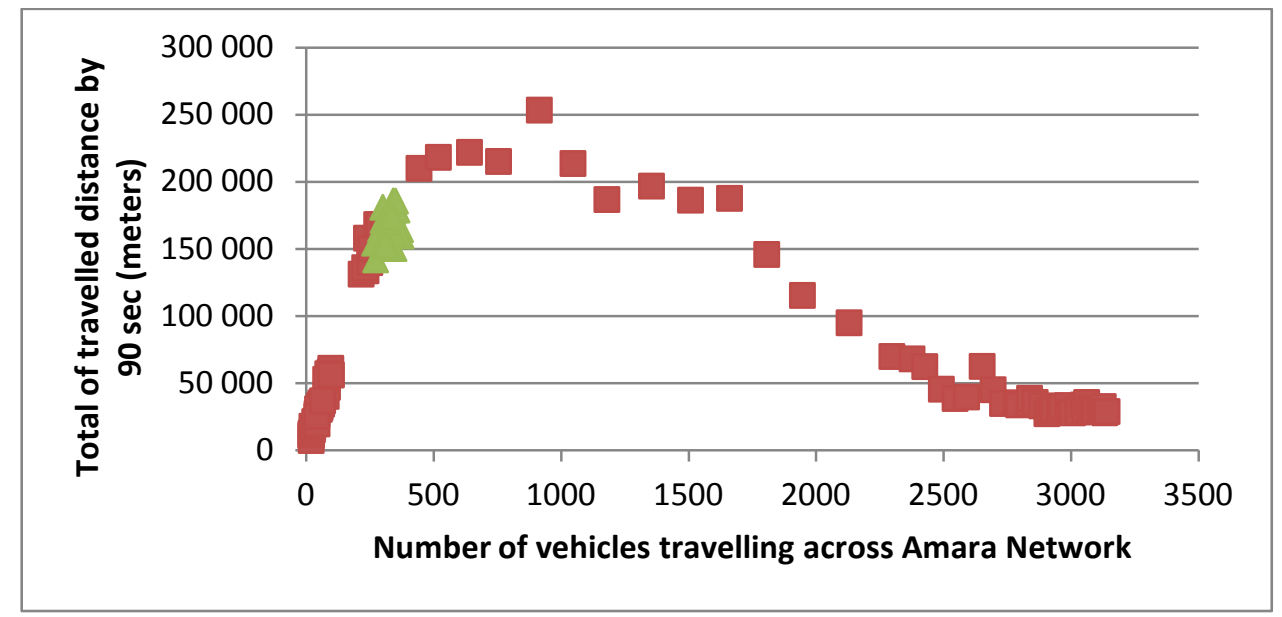

Figure 8. MFD AMARA Test Site: Historic OD Matrix (1h) (green triangles)

Figure 9 depicts the methodological framework for the simulation experiments. The assumed OD matrix is the result of applying some perturbation to the true historical matrix.

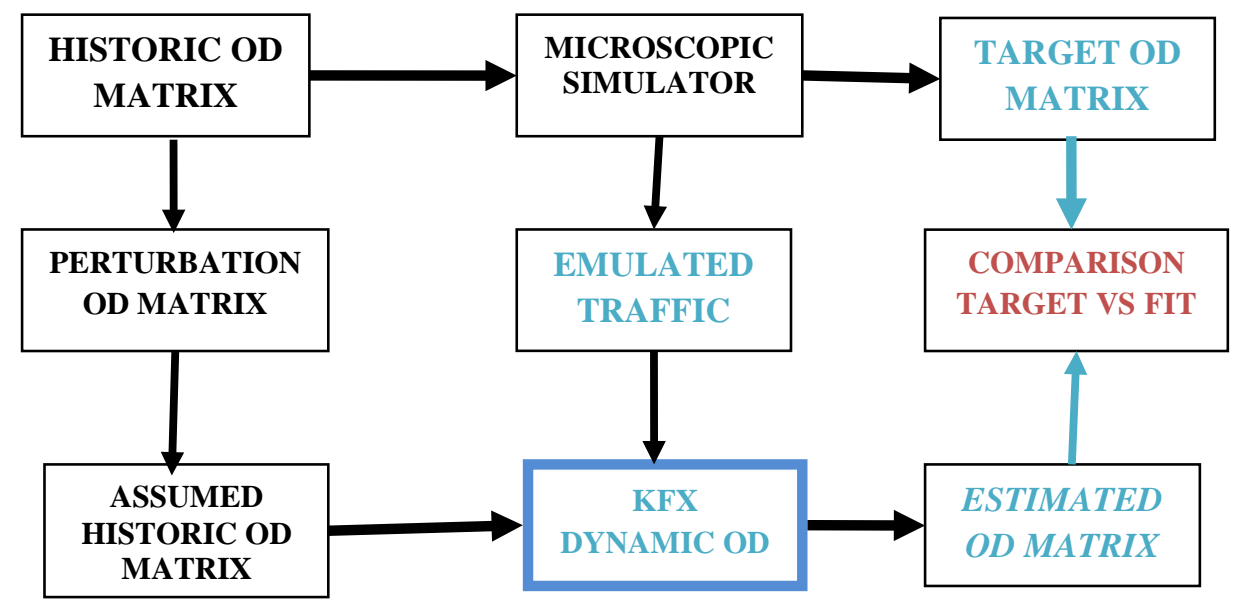

Figure 9. Methodological Design Of The Computational Experiment By Simulation

Tuning parameters (not considered as design factors) are:

- Deviate tuning parameter $\left(v_{1}\right)$ : scalar value affecting the historic OD flows for generating the assumed historic OD flows for deviate setting. Its value is set to 1 if the historic OD flows are reliable, otherwise increase/decrease.

- Interval length $\Delta t$. ( $\Delta t=90$ seconds has been used in this paper)

Design factors considered in computational experiments are:

Factor 1. The initialization of the state variables $\Delta g_{i j c}(0)$ is set to 0 . That is, OD path flows are equal to the assumed historical OD path flows for all time intervals, and the OD 
paths are the most likely used paths from a DUE assignment. The levels of Factor 1 depend on the generation of the assumed historical OD, according to whether:

a) It is generated by a perturbation of the historical OD by the $v_{1}$ tuning parameter and the OD pattern is preserved. The path proportions for each OD pair are taken as constant and equal.

b) It is generated by a perturbation of the historical OD by the $v_{1}$ tuning parameter but the OD pattern is not preserved. A subset $C$ of origin centroids is selected in terms of the total demand, partitioning the set $\mathfrak{I}$ of OD pairs as $\mathfrak{I}=\mathfrak{I}_{C} \cup\left\{\mathfrak{I} \mid \mathfrak{I}_{C}\right\}$, where $\mathfrak{I}_{C}$ is the set of OD pairs associated with C. Path proportions for OD pairs in $\left\{\mathfrak{I} \mid \mathfrak{I}_{C}\right\}$ follow the original OD pattern as in (a), while path proportions for OD pairs in $\mathfrak{I}_{C}$ are those in (a) multiplied by a factor that depends on $\left|\mathfrak{I}_{\mathrm{D} \mid \mathrm{i}}\right|$, the number of destinations that can be reached from origin $\mathrm{i} \in \mathrm{C}$. The level of perturbation is determined by $|C|=\{0,1,2,3 \ldots I\}$, where $C=\varnothing$ corresponds to case (a).

Factor 2. The variable percentage of ICT equipped vehicles, with levels of $20 \%$, 30\%, $50 \%$ and $100 \%$.

Factor 3. Diagonal and Multinomial structures for var-cov matrices of state variables. Only the diagonal structure applies for var-cov measurements in the current implementation.

Factor 4. Number $\mathrm{H}$ of bins used for the adaptive discrete approximations of travel time distributions (time-varying model parameters). $\mathrm{H}=3$ and 5 have been tested.

Factor 5. The dimension $\operatorname{IJKx}(\mathrm{M}+1)$ of the extended state vector. The values $\mathrm{M}=5,10$ have been considered.

Factor 6. Tolerance to conservation of entry flows at origins has been explicitly considered as measure equations with near-zero variance if tolerance is set to 0 .

Critical factors have proved to be Factors 1, 2 and 3; mainly the first two.

\section{Collected Performance Indicators}

Target OD flows per interval are compared with estimated OD flows (filtered OD flows) per interval at OD pair level by means of Theil's coefficient. Theil's inequality coefficient is a measurement of how close two time series are; bounded between 0 and $1, \mathrm{U}=0$ can be interpreted as a perfect fitting between the two series, while $U=1$ represents an unacceptable discrepancy.

Values of $U>0.2$ recommend rejecting the fit. Theil's coefficient (U) can be computed for each OD pair or for a subset of OD pairs (i.e., defined by the quantiles of hourly historical OD flows). If all feasible OD pairs are considered, then a global Theil measure of fit, GU, is computed as:

$$
G U=\frac{\sqrt{\frac{1}{I J \cdot G} \sum_{k=1: G} \sum_{o d=1: I J}\left(y_{o d, k}-\hat{y}_{o d, k}\right)^{2}}}{\sqrt{\frac{1}{I J \cdot G} \sum_{k=1: G} \sum_{o d=1: I J} \hat{y}_{o d, g}{ }^{2}}+\sqrt{\frac{1}{I J \cdot G} \sum_{k=1: G} \sum_{o d=1: I J} y_{o d, k}{ }^{2}}}
$$

Other performance indicators, used either for individual OD pairs or groups of OD pairs, ,are the normalized root mean square error (RMSEN), a weighted indicator for subsets of OD pairs (usually a subset of OD pairs whose hourly flow is at 25\% of higher flows), and a weighted global indicator for the whole set of OD pairs (GRMSEN, sum of squared 
differences between target and estimated path flows per interval, relative to total target flows during the simulation horizon):

$$
R M S E N=\frac{\sqrt{G \sum\left(y_{k}-\hat{y}_{k}\right)^{2}}}{\sum y_{k}} \quad G R M S E N=\frac{\sqrt{I J \cdot G \sum_{k=1: G} \sum_{o d=1: I J}\left(y_{o d, k}-\hat{y}_{o d, k}\right)^{2}}}{\sum_{k=1: G} \sum_{o d=1: I J} y_{o d, k}}
$$

\section{COMPUTATIONAL RESULTS FOR AMARA TEST SITE}

Microscopic simulation with AIMSUN induces variability in the historical inputs in a realistic way and produces target OD flows per interval (related to the true historical OD matrix).

Figure 10 visualizes the results for the two most relevant OD flows when the base initialization is selected for Factor 1, i.e., the assumed historical OD matrix is reliable. The concordance is numerically quantified in terms of RMSEN and U for OD flows from origins 765 and 768 to all destinations in the network in Table 4. The low values of RMSEN and U prove good behavior of KFX for OD pairs with large demand (those in the $4^{\text {th }}$ quartile of OD flows).

Theil's coefficients are 0.11 and 0.10 , respectively, for the selected OD pairs, and GU is 0.15 . Small OD flows show worse behavior, due to few observations per interval and, thus, tuning parameter $\Delta$ t should be increased to properly deal with the sparse reality. Although not shown in Table 4, we studied the effect of decreasing the penetration rates of BT technology (Factor 2) in the selected OD pairs, showing an increase in indicators (U, RMSEN) and, thus, a decrease in the quality of the results as equipped rate decreases (after controlling Factor 1 and 3 to 6). So, the decrease in BT penetration means fewer observations and larger $\Delta$ t needed for practical purposes.
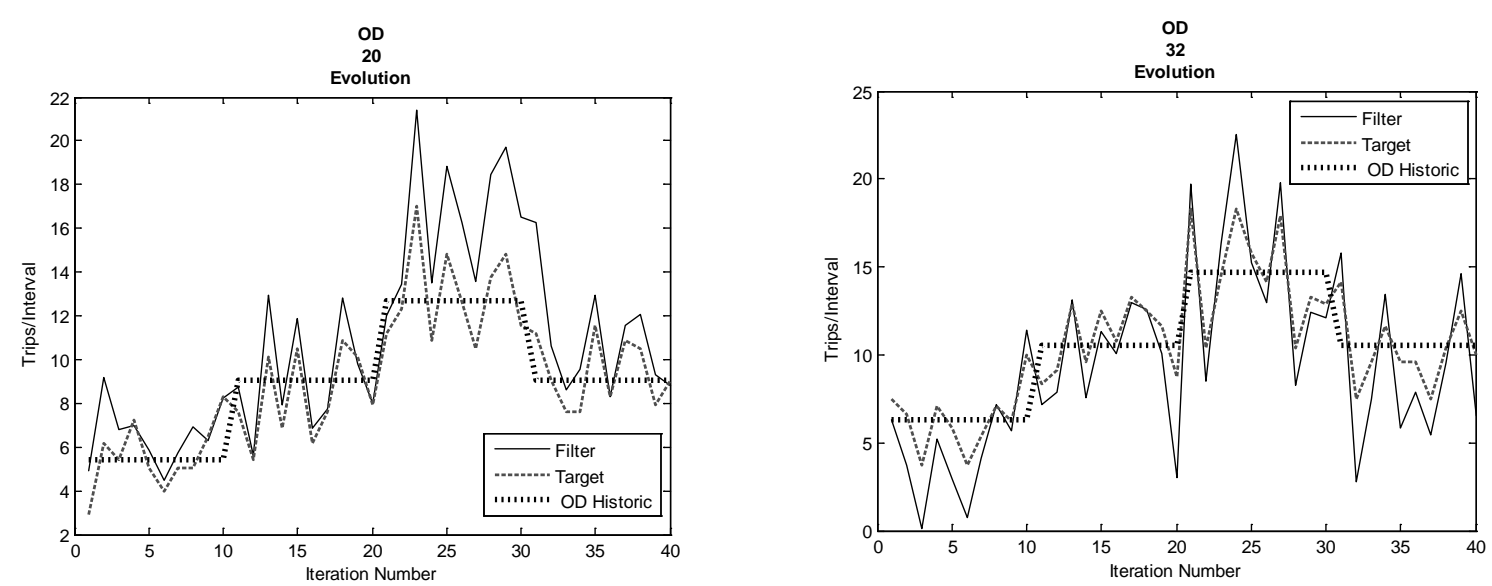

(a) (b)

Figure 10. (100\% Equipped Vehicles): Filtered OD Flows, Target OD Flows And Historic OD Flows For OD-Pair 20.765-777 (a) and OD-pair 32.768-783 (b).

The aggregated hourly fit between estimated KFX versus Target OD flows shows a regression R squared higher to $90 \%$ (see Figure 11). In $100 \%$ of equipped vehicles, the results show that the joint effects of factors 4, 5 and 6 are irrelevant: more than $\mathrm{H}=3$ bins (Factor 4) are not necessary. Results do not improve substantially if the number of time intervals $M$ to define the extended state vector is increased from 5 to $\mathrm{M}=10$ (Factor 5) intervals, since $\mathrm{M}=5$ accounts for more than $90 \%$ of the OD travel times and dimensions of the state vector is 
divided by 2. There are some OD pairs with low demand in Table 4 that exhibit a complete lack of convergence according to Theil's coefficient $U$, since few observations per interval are present (OD pairs in the $1^{\text {st }}$ and $2^{\text {nd }}$ quantile, according to flow magnitudes).

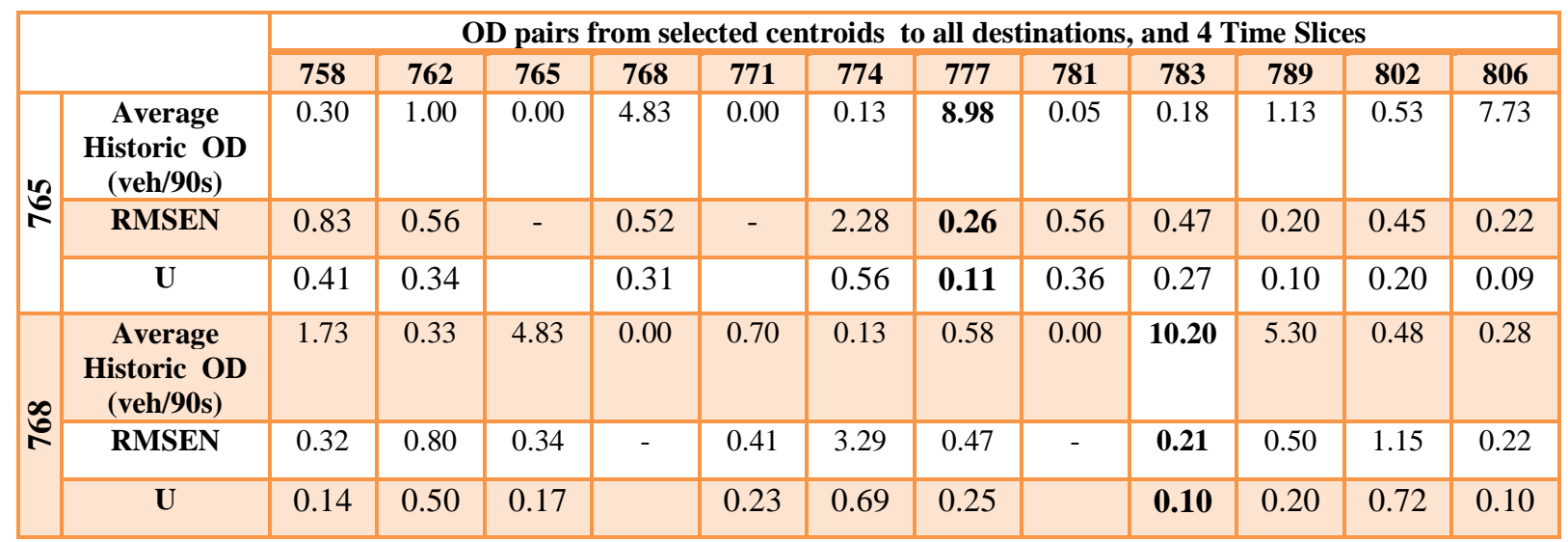

Table 4. OD Pairs From Selected Centroids To All Destinations, and 4 Time Slices For The Base Initialization And $100 \%$ Of Equipped Vehicles

Since OD path flows are not restricted to being positive, negative OD path flows might occur for low demand OD pairs. However, in the aggregation process of obtaining OD pair flows, no negative values have been encountered. Considering the 4th quantile OD flows, we tested how the assumed Historical OD path flows affect KFX convergence in 100\% of equipped vehicles (Factor 2) and a diagonal structure for var-cov matrices (Factor 3) (see Table 5).

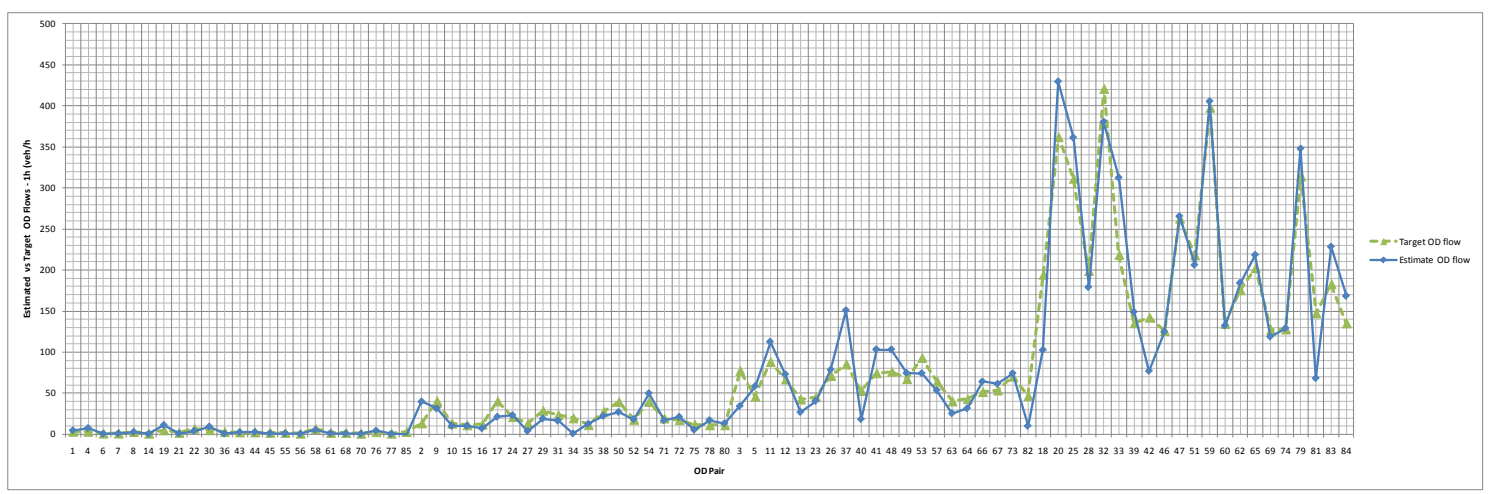

Figure 11. Estimated (KF approach) vs Target flows in OD pairs for the base initialization and $100 \%$ of equipped vehicles

For practical purposes, Factor 1 is critical. When controlling Factor 2 to $100 \%$ equipped (not realistic), the historical matrix reliability does not seriously affect the global results (GU and GRMSEN): even in the most uninformative assumed historical OD flows, GRMSEN stays around 0.3 , considering 25\% of the most important OD pairs, and is reduced to 0.16 and 0.2 for pairs 20 and 32 (the largest ones). In terms of serial matching for the overall $4^{\text {th }}$ quantile OD flows, the effect of a bad input is minor: GU never increases to 0.20 but, for the most important pairs 20 and 32, $\mathrm{U}$ values range from 0.07 to 0.17 (excellent coefficients). The behavior is very clear: OD pairs with large flows converge to correct values but, when sparse observations are available, no correction for a priori assumed flows is possible and, thus, no convergence is achieved (for OD pairs in the $1^{\text {st }}$ and $2^{\text {nd }}$ quantile of OD flows, GU for them ranges from 0.28 to 0.74 ). Thus, OD pairs with large volumes are not seriously affected by the 
quality of the historical matrix, which exhibits good convergence properties that are needed for practical applications.

In Table 5, in the columns related to $100 \%$ BT penetration, aggregated results for the whole set of OD pairs and for 25\% of the most important OD pairs are detailed. Comparisons are based on GRMSEN, GU and $\mathrm{R}^{2}$ indicators. Thus, even with the worst input, the overall GU is 0.42 and GRMSEN is $502 \%$. But $25 \%$ of the larger OD pairs exhibit values of 0.16 and $31 \%$, respectively.

\begin{tabular}{|c|c|c|c|c|c|c|c|c|c|c|c|c|}
\hline \multirow{3}{*}{$\begin{array}{l}\text { Factor 1 - } \\
\text { Perturbation } \\
\text { to historic } \\
\text { OD pattern }\end{array}$} & \multicolumn{12}{|c|}{$\begin{array}{l}\text { Factor } 1 \text { - Global Theil Coefficient (GU) and RMSEN and } R^{2} \text { for percentage of ICT equipped } \\
\text { vehicles (Factor 2). Indicators for 4th Quantile OD flows in parenthesis. }\end{array}$} \\
\hline & \multicolumn{3}{|c|}{$20 \%$} & \multicolumn{3}{|c|}{$30 \%$} & \multicolumn{3}{|c|}{$50 \%$} & \multicolumn{3}{|c|}{$100 \%$} \\
\hline & GU & GRMSEN & $\mathbf{R}^{2}$ & GU C & GRMSEN & $\mathbf{R}^{2}$ & GU & GRMSEN & $\mathbf{R}^{2}$ & GU & GRMSEN & $\mathbf{R}^{2}$ \\
\hline 0-none & $\begin{array}{c}0.34 \\
(0.23)\end{array}$ & $\begin{array}{c}1.04 \\
(0.53)\end{array}$ & $88.9 \%$ & $\begin{array}{c}0.31 \\
(0.19)\end{array}$ & $\begin{array}{c}0.88 \\
(0.43)\end{array}$ & $92 \%$ & $\begin{array}{c}0.30 \\
(0.17)\end{array}$ & $\begin{array}{c}0.74 \\
(0.37)\end{array}$ & 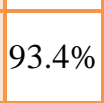 & $\begin{array}{c}0.27 \\
(0.15)\end{array}$ & $\begin{array}{c}0.63 \\
(0.31)\end{array}$ & $93.9 \%$ \\
\hline 1 & $\begin{array}{c}0.36 \\
(0.23)\end{array}$ & $\begin{array}{c}1.44 \\
(0.52)\end{array}$ & & $\begin{array}{c}0.34 \\
(0.19)\end{array}$ & $\begin{array}{c}1.29 \\
(0.42)\end{array}$ & 91.1\% & $\begin{array}{l}0.32 \\
(0.17)\end{array}$ & $\begin{array}{c}1.21 \\
(0.36)\end{array}$ & & $\begin{array}{c}0.25 \\
(0.15)\end{array}$ & $\begin{array}{c}0.93 \\
(0.30)\end{array}$ & $92.9 \%$ \\
\hline 2 & $\begin{array}{c}0.36 \\
(0.23)\end{array}$ & $\begin{array}{c}1.47 \\
(0.51)\end{array}$ & $87.2 \%$ & $\begin{array}{c}0.34 \\
(0.19)\end{array}$ & $\begin{array}{c}1.31 \\
(0.42) \\
\end{array}$ & $91 \%$ & $\begin{array}{c}0.32 \\
(0.17)\end{array}$ & $\begin{array}{c}1.20 \\
(0.36)\end{array}$ & & $\begin{array}{l}0.26 \\
(0.15)\end{array}$ & $\begin{array}{c}0.95 \\
(0.30) \\
\end{array}$ & 92.9\% \\
\hline 3 & $\begin{array}{c}0.38 \\
(0.23)\end{array}$ & $\begin{array}{c}2.17 \\
(0.51)\end{array}$ & 840 & $(0.37)$ & $\begin{array}{c}2.04 \\
(0.42)\end{array}$ & $88 \mathrm{c}$ & $\begin{array}{l}0.35 \\
(0.17)\end{array}$ & $\begin{array}{c}1.94 \\
(0.36)\end{array}$ & 90. & $\begin{array}{c}0.28 \\
(0.15)\end{array}$ & $\begin{array}{c}1.68 \\
(0.31)\end{array}$ & $90.7 \%$ \\
\hline all & $\begin{array}{c}0.45 \\
(0.24)\end{array}$ & $\begin{array}{c}5.21 \\
(0.53)\end{array}$ & $76.9 \%$ & $\left(\begin{array}{c}0.43 \\
(0.20)\end{array}\right.$ & $\begin{array}{c}4.97 \\
(0.42)\end{array}$ & $81.8 \%$ & $\begin{array}{c}0.42 \\
(0.18)\end{array}$ & $\begin{array}{c}4.99 \\
(0.37)\end{array}$ & 82 & $\begin{array}{c}0.42 \\
(0.16)\end{array}$ & $\begin{array}{c}5.02 \\
(0.31)\end{array}$ & 81.7\% \\
\hline
\end{tabular}

Table 5. GU, GRMSEN, $\mathbf{R}^{2}$ According To Design Factors 1 And 2 (Quality Of The Assumed Historical OD Matrix And Percentage Of ICT Equipped Vehicles)

When facing Factor 2, i.e., BT penetration rates, the experiments show that when the proportion of equipped vehicles decreases, fewer observations of vehicles through sensors are available for feeding KFX; what's more, the global indicators GU and GRMSEN increase while $\mathrm{R}^{2}$ decreases. Therefore, the dynamic estimation of OD matrices worsens. This behavior is shown in Table 5 by the effect of Factor 2 (percentage of ICT equipped vehicles). In the case of a reliable OD matrix (Factor 1 - level 0 ), for $25 \%$ of the most important OD pairs, GU increases from 0.15 (100\% penetration) to 0.23 (20\% penetration) when all other design factors are kept constant. But in the case of a non-reliable assumed matrix (Factor 1 level - all, shown in the last row of Table 5), GU increases from 0.16 (100\% penetration) to 0.24 (20\% penetration) for the $25 \%$ most important OD pairs. This is nearly non-sensible, in terms of a badly assumed OD matrix. But for the global set of OD pairs, GU ranges from 0.42-0.45, regardless of the percentage of equipped vehicles (again, shown in the last row of Table 5). Meanwhile, within a good assumed matrix (first row Table 5), the global GU for the whole set of OD pairs is 0.27 in $100 \%$ of equipped vehicles, but 0.34 in under $20 \%$ of equipped vehicles. So, important OD pairs are affected by the percentage of equipped vehicles much more than by the quality of the assumed matrix.

The applicability to a real test-site is clear: since the length of the time interval is critical, it should be the minimum length of time that is able to capture the most important OD pair relations with the actual percentage of equipped vehicles. This happens when non-null registers of observations for equipped vehicles per interval are available. The trade-off interval length and equipped counts has to be carefully addressed. 
A few comments on Factor 3: the structure of the variance-covariance matrix for the extended state variables (OD path flows in M+1 time intervals) behaves very differently in KFX. KFX tuning parameters are highly dependent on variance-covariance structure. In the absence of reliable OD historical patterns, a diagonal var-cov proposal is recommended, since it adapts more easily and converges faster. But performance indicators are globally more stable across Factor 2 levels, when a multinomial structure is selected. Further work is needed on Factor 3.

\section{APPLICATION TO FREEWAYS: RONDA DE DALT SIMULATION SITE}

An urban freeway in Barcelona has been selected as a second test site --a 11.551-km-long section of the Ronda de Dalt-- between the Trinitat and the Diagonal Exchange Nodes. The site has 11 entry ramps and 12 exit ramps (including main section flows) on the section being studied, which flows in the direction of Llobregat (to the south of the city). Figure 12 depicts a part of the site with the suggested sensor layout. $\mathrm{D}_{\mathrm{i}}$ denotes the location of the $\mathrm{i}$-th sensor at the main section; $E_{j}$ denotes the sensor located at the $j$-th entry ramp and $S_{n}$ the sensor located at the n-th exit ramp. State variables are OD path flows, but since there is just one path for each OD pair, state variables are direct OD pair flows.
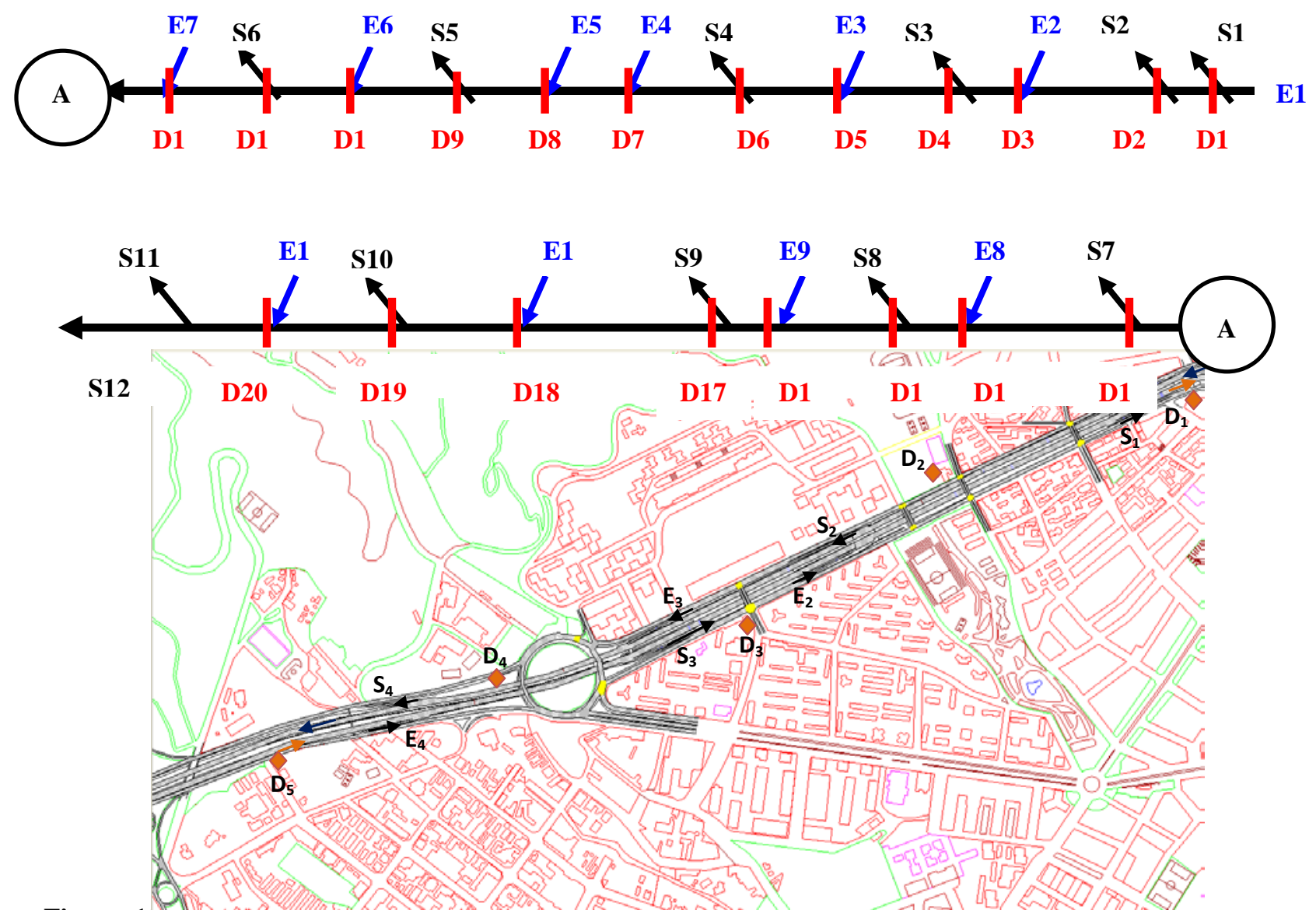

Figure 12. A segment of the site tor the UD estimation showing part of the detection layout and diagram with the conceptual structure

A set of computational experiments has been conducted with simulated data, with the aim of testing the influence of the quality of the Historic OD data and the percentage of equipped vehicles (design factors 1 and 2). The methodological design of the computational experiments by simulation follows Figure 9. There are 74 OD pairs and OD path flows because, in the freeway site model, a unique path is associated to each OD pair. The most important entries are 1, 2 and 3; but mostly 1, since most of the vehicles go from the test site's 
first entrance (mainstream) through to the last freeway test site segment. R-squared fit would not be a good indicator, due to the presence of such an influence from OD pairs 1-12.

Time horizon is defined as 100 minutes, and a 150-second subinterval time length is considered. A true historic OD pattern which is representative of an average morning peak period has been selected. Time variable demand has been split into 4 time-slices (with fixed OD pattern) accounting for $15 \%, 25 \%, 35 \%$ and $25 \%$ of the total demand in each 25 -minute slice. Demand has been defined according to the MFD methodology, accounting for a level of demand which rises to the point of congestion.

According to previous experience, an extended state vector of at least 7 subintervals plus the current one would be enough, since it contains $90 \%$ of the trip travel times. But $\mathrm{M}$ is set to 12 , since no massive computations are required for this low dimension test. The number of intervals for the discretization of travel time distributions is set to $\mathrm{H}=5$.

\section{COMPUTATIONAL RESULTS FOR THE FREEWAY SIMULATION SITE}

Results included in this paper affect Factor 1 (quality of the assumed historic OD flows for computing deviates as state variables) and Factor 2 (Percentage of equipped vehicles) with levels 20\%, 30\%, 50\% and 100\% The tests show that the proposed Kalman Filtering approach converges successfully to the simulated OD flows in a few iterations for $100 \%$ of equipped vehicles (Factor 2). Also, the assumed historical OD pattern is preserved as the true historical OD pattern for all the entries to the freeway. For all OD pairs on a global level, the global fit decreases slightly (GU and GRMSEN in Table 6) as the percentage of equipped vehicles decreases (Factor 2). However, the global fit is much more sensitive to the quality of the assumed historical information provided (Factor 1), since OD pairs with low flows do not converge. Still, the quality of the assumed historical information does not seriously affect the fit for OD pairs with high flows.

\begin{tabular}{|c|c|c|c|c|c|c|c|c|c|c|c|c|}
\hline \multirow{3}{*}{\begin{tabular}{|r|} 
Factor 1- \\
Perturbation \\
to historic \\
OD pattern
\end{tabular}} & \multicolumn{12}{|c|}{$\begin{array}{l}\text { Global Theil Coefficient (GU) and RMSEN and } R^{2} \text { for percentage of ICT equipped vehicles } \\
\text { (Factor 2). Indicators for 4th Quantile OD flows in parenthesis }\end{array}$} \\
\hline & \multicolumn{3}{|c|}{$20 \%$} & \multicolumn{3}{|c|}{$30 \%$} & \multicolumn{3}{|c|}{$50 \%$} & \multicolumn{3}{|c|}{$100 \%$} \\
\hline & GU & GRMSEN & $\mathbf{R}^{2}$ & GU & GRMSEN & $\mathbf{R}^{2}$ & GU & GRMSEN & $\mathbf{R}^{2}$ & GU & GRMSEN & $\mathbf{R}^{2}$ \\
\hline 0-none & $\begin{array}{c}0.22 \\
(0.11)\end{array}$ & $\begin{array}{c}0.39 \\
(0.24)\end{array}$ & $99.7 \%$ & $\begin{array}{c}0.20 \\
(0.10)\end{array}$ & $\begin{array}{c}0.33 \\
(0.22)\end{array}$ & $99.7 \%$ & $\begin{array}{c}0.18 \\
(0.09)\end{array}$ & $\begin{array}{c}0.29 \\
(0.19)\end{array}$ & $99.7 \%$ & $\begin{array}{c}0.17 \\
(0.09)\end{array}$ & $\begin{array}{c}0.25 \\
(0.18)\end{array}$ & $99.7 \%$ \\
\hline 1 & $\begin{array}{c}0.24 \\
(0.12)\end{array}$ & \begin{tabular}{|c|}
0.43 \\
$(0.29)$
\end{tabular} & $99.8 \%($ & $\begin{array}{c}0.22 \\
(0.11)\end{array}$ & $\begin{array}{c}0.37 \\
(0.26)\end{array}$ & $99.8 \%$ & $\begin{array}{c}0.21 \\
(0.10)\end{array}$ & $\begin{array}{c}0.32 \\
(0.24)\end{array}$ & $99.7 \%$ & $\begin{array}{c}0.19 \\
(0.10)\end{array}$ & $\begin{array}{c}0.29 \\
(0.23)\end{array}$ & $99.7 \%$ \\
\hline 2 & $\begin{array}{c}0.25 \\
(0.11)\end{array}$ & \begin{tabular}{|c|}
0.43 \\
$(0.28)$
\end{tabular} & $99.8 \%($ & $\begin{array}{c}0.23 \\
(0.10)\end{array}$ & $\begin{array}{c}0.36 \\
(0.25)\end{array}$ & $99.8 \%$ & $\begin{array}{c}0.21 \\
(0.10)\end{array}$ & $\begin{array}{c}0.32 \\
(0.23)\end{array}$ & 99.8\% & $\begin{array}{c}0.20 \\
(0.09)\end{array}$ & $\begin{array}{c}0.28 \\
(0.22)\end{array}$ & $99.8 \%$ \\
\hline 3 & $\begin{array}{c}0.27 \\
(0.11)\end{array}$ & $\begin{array}{c}0.44 \\
(0.27)\end{array}$ & $99.8 \%($ & $\begin{array}{c}0.25 \\
(0.10)\end{array}$ & $\begin{array}{c}0.37 \\
(0.24)\end{array}$ & $99.8 \%$ & $\begin{array}{c}0.23 \\
(0.10)\end{array}$ & $\begin{array}{c}0.34 \\
(0.22)\end{array}$ & 99.8\% & $\begin{array}{c}0.22 \\
(0.09)\end{array}$ & $\begin{array}{c}0.30 \\
(0.21)\end{array}$ & $99.8 \%$ \\
\hline all & $\begin{array}{c}0.40 \\
(0.14)\end{array}$ & $\begin{array}{c}1.40 \\
(0.34)\end{array}$ & $99.7 \%($ & $\begin{array}{c}0.39 \\
(0.14)\end{array}$ & $\begin{array}{c}1.29 \\
(0.31)\end{array}$ & $99.7 \%$ & $\begin{array}{c}0.39 \\
(0.13)\end{array}$ & $\begin{array}{c}1.26 \\
(0.29)\end{array}$ & 99.7\% & $\begin{array}{c}0.38 \\
(0.13)\end{array}$ & $\begin{array}{c}1.17 \\
(0.27)\end{array}$ & $99.7 \%$ \\
\hline
\end{tabular}

Table 6. Freeway test: Global Theil Coefficient (GU), RMSEN and $\mathbf{R}^{2}$ according to Design Factors 1 and 2 (Percentage of ICT equipped vehicles) 


\section{CONCLUSIONS}

This paper summarizes the results of research into the potential advantages of combining traffic measurements provided by the usual technologies (e.g. inductive loop detectors) with those supplied by the applications of ICT technologies. We have focused our attention on Bluetooth sensors, which are capable of uniquely identifying onboard Bluetooth devices when measuring travel times, although they could be replaced by any ICT application with similar detection functionalities. The raw measurements from Bluetooth sensors cannot be used directly; so, a specific filtering technique was successfully developed and tested. The clean data was then used as input for the OD estimation. The main assumption was that these type of measurements allow an ad hoc Kalman Filter estimate of time-dependent OD matrices. This can maintain the usual linear structure because the ICT measurements can replace the necessary information usually generated by nonlinear models in the Extended Kalman Filter. The computational experiments confirm the validity of this hypothesis and the reduction of the computational requirements open the door to the possibility of using this approach for real time applications. Taking into account that has been implemented in Matlab, it is plausible that migration to $\mathrm{C}++$ could significantly reduce the computing requirements, even for larger networks. The new approach has been successfully tested by simulation in two different scenarios: freeways and small urban networks. In the urban case, the formulation takes into account the network topology, i.e., multiple paths between OD pairs that, according to a DUE model, are the most likely used paths. The strategy of using the DUE most likely used paths to define the KFX state variables looks promising, in view of the computational results, since time-dependent path proportion shares and assignment matrices are not employed in the formulation or as input data. The application of this OD estimation method to urban networks raises a question on the suitability of the detection layout for OD estimation. A special model has been developed and used in the synthetic simulation experiments. The model is based on the characteristics of Bluetooth detection and the condition of using this technology. The results obtained have confirmed the validity of the hypothesis and fostered new research into the robustness of the proposed method, its sensitivity to changes in the design factors, and the sizes of the networks to which it could still be applied in real time.

\section{ACKNOWLEDGEMENTS}

This research is funded by projects SIMETRIA (Ref. P 63/08, 27.11.2008), MITRA (TRA2009-14270) and TRA2008-06782-C02-02 of the Spanish R+D National Programs and has benefited from participation in EU COST Action TU0903 MULTITUDE - Methods and tools for supporting the Use caLibration and validaTIon of Traffic simUlation models.

\section{REFERENCES}

Antoniou, C., Ben-Akiva, M. \& Koutsopoulos H.N. (2007). Nonlinear Kalman Filtering Algorithms for On-Line Calibration of Dynamic Traffic Assignment Models, IEEE Transactions on Intelligent Transportation Systems Vol. 8 No.4.

Ashok, K. \& Ben-Akiva, M. (1993). Dynamic origin-destination matrix estimation for realtime traffic management systems. In Transportation and Traffic Theory, C.F. Daganzo (Ed.), Elsevier Science Publishers, Oxford, UK, 465-484.

Ashok, K. \& Ben-Akiva, M. (2000). Alternative Approaches for Real-Time Estimation and Prediction of Time-Dependent Origin-Destination Flows, Transportation Science 34(1), 2136. 
Barceló J.,.Montero L, Marqués L. \& Carmona C. (2010a). Travel time forecasting and dynamic of estimation in freeways based on Bluetooth traffic monitoring, Transportation Research Records: Journal of the Transportation Research Board, Vol. 2175, 19-27.

Barceló J., Montero L., Marqués L. \& Carmona C. (2010b). A Kalman-Filter Approach For Dynamic OD Estimation In Corridors Based On Bluetooth And Wifi Data Collection, Paper number 01387, presented at the $12^{\text {th }}$ World Conference on Transportation Research, Lisbon July 11-15, included in the CD-Rom.

Barceló J., Gilliéron F., Linares M.P., Serch O. \& Montero L. (2012). The Detection Layout Problem, Paper \#12-2056 presented at the $91^{\text {st }}$ Annual TRB Meeting and accepted to be published in Transportation Research Record.

Cascetta, E. (1984). Estimators of trip matrices from traffic counts and survey data: a generalized least squares estimator, Transportation Research 18B (4/5), 289-299.

Castillo, E. Conejo, A. J. Menéndez, J. M. \& Jiménez, P. (2008). The Observability Problem in Traffic Network Models, Computer-Aided Civil and Infrastructure Engineering 23, 208222

Chang, G.L. \& Wu, J. (1994). Recursive estimation of time-varying Origin-Destination flows from traffic counts in freeway corridors, Transportation Research B Volume 28B No.2, 141-160.

Choi, K., Jayakrishnan, R., Kim, H., Yang, I. \& Lee, J. (2009). Dynamic OD Estimation using Dynamic Traffic Simulation Model in an Urban Arterial Corridor, Paper presented the $88^{\mathrm{th}}$ TRB Meeting, Washington D.C.

Codina E. \& Barceló, J. (2004). Adjustment of O-D matrices from observed volumes: an algorithmic approach based on conjugate gradients, European Journal of Operations Research Vol. 155, pp. 535-557.

Daganzo, C. F. \& N. Geroliminis (2008). An analytical approximation for the macroscopic fundamental diagram of urban traffic, Transportation Research B Volume 42 (9), 759-770.

Dixon, M.P \& Rilett, L.R. (2002). Real-Time OD Estimation using Automatic Vehicle Identification and Traffic Count data, Computer-Aided Civil and Infrastructure Engineering, Vol 17 7-21.

Dynameq (2010). Version 1.4.6, INRO www.inro.ca.

Florian, M., Mahut, M. \& Tremblay, N. (2001). A Hybrid Optimization-Mesoscopic Simulation Dynamic Traffic Assignment Model, Proceedings of the 2001 IEEE Intelligent Transport Systems Conference, Oakland, 118-123.

Gilliéron F. \& Barceló J. (2008). Optimization of road traffic counts location in a network, Paper 2728 presented at the ITS 2008, European ITS Conference, Genève, included in the Conference CD-ROM.

Hu, S., Madanat S.M., Krogmeier J. \& Peeta S. (2001). Estimation of Dynamic Assignment Matrices and OD Demands using Adaptative Kalman filtering, Intelligent Transportation Systems Journal vol. 6, pp 281-300. 
Kalman, R.E. (1960). A new approach to linear filtering and prediction problem, Journal of Basic Engineering 82 (1), 35-45.

Lin, P. \& Chang, G. (2007). A generalized model and solution algorithm for estimation of the dynamic freeway origin-destination matrix, Transportation Research B 41 554-572.

Lundgren J. T. \& Peterson A. (2008). A heuristic for the bilevel origin-destination-matrix estimation problem, Transportation Research Part B 42 339-354.

Ran, B., Jin, P.J., Boyce, D., Qiu, T.Z. \& Cheng, Y. (2012). Perspectives on Future Transportation Research: Impact of Intelligent Transportation System Technologies on NextGeneration Transportation Modeling, Journal of Intelligent Transportation Systems Vol. 16, Iss. 4.

Treiber M., Kesting A. \& Wilson R. E. (2011). Reconstructing the Traffic State by Fusion of Heterogeneous Data, Computer-Aided Civil and Infrastructure Engineering, 26, 408-419.

Van Der Zijpp, N.J. \& Hamerslag, R. (1994). An improved Kalman filtering approach to estimate Origin-Destination matrices for freeway corridors, Transportation Research Records 1443, 54-64.

Van Lint J.W.C. \& Hoogendoorn S. P. (2009). A Robust and Efficient Method for Fusing Heterogeneous Data from Traffic Sensors on Freeways, Computer-Aided Civil and Infrastructure Engineering 24, 1-17.

Van Zuylen, J. H. \& Willumsen, L. G. (1980). The most likely trip matrix estimated from traffic counts, Transportation Research 14B, 281-293.

Work, D., Toossavainen, O-P., Blandin, S., Bayen, A., Iwuchukwu, T. \& Tracton, K. (2008). An Ensemble Kalman Filtering Approach to Highway Traffic Estimation Using GPS Enabled Mobile Devices, Proceedings of the 47th IEEE Conference on Decision and Control, 50625068, Cancun, Mexico, Dec. 9-11, 2008, DOI: 10.1109/CDC.2008.4739016.

Yang, H. \& Zhou, J. (1998). Optimal traffic counting locations for origin-destination matrix estimation, Transportation Research, 32B, 109-26.

Zhou, X. \& Mahmassani, H.S. (2006). Dynamic origin-destination demand estimation using automatic vehicle identification data, IEEE Transactions on Intelligent Transportation Systems Vol 7, $105-114$. 